\title{
QUANTITATIVE EXTENSIONS OF PLURICANONICAL FORMS AND CLOSED POSITIVE CURRENTS
}

\author{
BO BERNDTSSON AND MIHAI PĂUN
}

\begin{abstract}
We establish here several "invariance of plurigenera type" theorems for twisted pluricanonical forms and metrics of adjoint $\mathbb{R}$-bundles.
\end{abstract}

\section{$\S 0$. Introduction}

In this article, we are mainly concerned with the extension properties of twisted pluricanonical forms. Part of the motivation for this work has its origins in algebraic geometry. Indeed, many of the techniques that we generalize and refine here have been successfully used to solve important problems, such as boundedness of pluricanonical maps of varieties of general type and finiteness of the algebra associated to the canonical ring.

Let $\mathcal{X} \rightarrow \mathbb{D}$ be a smooth and projective family of complex manifolds over the unit disk $\mathbb{D}$, and let $\left(F, h_{F}\right) \rightarrow \mathcal{X}$ be a line bundle endowed with a metric $h_{F}$. By convention, unless explicitly stated otherwise, all the metrics in what follows are allowed to be singular. The "standard" curvature and restriction assumptions for the metric $h_{F}$ are as follows.

(C) The curvature current of $\left(F, h_{F}\right)$ is semipositive; that is, $\sqrt{-1} \Theta_{h_{F}}(F) \geq$ 0 .

(R) The restriction of the metric $h_{F}$ to the central fiber is well defined: $h_{F \mid \mathcal{X}_{0}} \not \equiv \infty$.

Under these circumstances, the extension theorem established in [26] (and subsequently developed in [1], [2], [9], [21], [24], [25], [30], [33]) states the following. There exists a purely numerical constant $C_{0}>0$ such that for any section $u$ of the bundle $K_{\mathcal{X}_{0}}+F_{\mid \mathcal{X}_{0}}$ which is $L^{2}$ with respect to $h_{F}$, that is,

$$
\int_{\mathcal{X}_{0}}|u|^{2} e^{-\varphi_{F}}<\infty
$$

Received June 3, 2010. Revised November 20, 2010. Accepted July 15, 2011.

2010 Mathematics Subject Classification. Primary 32Q15; Secondary 14D07, 14E99. 
there exists a section $U$ of the bundle $K_{\mathcal{X}}+F$ whose restriction to $\mathcal{X}_{0}$ is equal to $u$, and such that

$$
\int_{\mathcal{X}}|U|^{2} e^{-\varphi_{F}} \leq C_{0} \int_{\mathcal{X}_{0}}|u|^{2} e^{-\varphi_{F}} .
$$

The meaning of the word restriction above is that over the central fiber we have $U_{\mid \mathcal{X}_{0}}=u \wedge d \pi$.

We prove in this article similar effective extension statements for bundles of type

$$
p K_{\mathcal{X}}+F
$$

If $p \geq 2$, a first result to be mentioned is the invariance of plurigenera due to Siu ([30]), which completely elucidates the case of pluricanonical forms (i.e., without the additional twisting $F$ ). Indeed, it is possible (and not very complicated) to further refine the result in [30] and obtain the following statement: let $u$ be a holomorphic section of the bundle $p K_{\mathcal{X}_{0}}$; then there exists a section $U$ of the bundle $p K_{\mathcal{X}}$ whose restriction to $\mathcal{X}_{0}$ is equal to $u$, that is,

$$
U_{\mid \mathcal{X}_{0}}=u \wedge(d \pi)^{\otimes p}
$$

and such that

$$
\int_{\mathcal{X}}|U|^{\frac{2}{p}} \leq C_{0} \int_{\mathcal{X}_{0}}|u|^{\frac{2}{p}}
$$

where $C_{0}$ is the same constant as above.

Motivated by applications in algebraic geometry, one has to generalize these kinds of results for twisted pluricanonical forms, but in this setting, the optimal integrability conditions to be imposed are less clear; for example, replacing (1) with the natural $L^{2 / p}$ convergence is not enough (see the examples in [12], [17]).

We describe next the results we obtain in the present article.

To start with, we recall the following notion. Consider an ideal $\mathcal{I} \subset \mathcal{O}_{X}$ and a positive integer $k \geq 0$. We denote by $\overline{\mathcal{I}^{(k)}}$ the integral closure of the $k$ th power of $\mathcal{I}$ : it is the ideal constructed as follows. Let $x \in X$ be an arbitrary point, and let $\left(f_{1}, \ldots, f_{r}\right)$ be the generators of $\mathcal{I}$ at $(X, x)$; we have

$$
\overline{\mathcal{I}}_{x}^{(k)}:=\left\{g \in \mathcal{O}_{X, x} /|g|^{\frac{2}{k}} \leq C \sum_{\alpha}\left|f_{\alpha}\right|^{2} \text { for some constant } C>0\right\} .
$$

In this context, we first prove the next refined version of the twisted invariance of plurigenera. 
TheOREM 0.1. Let $\pi: \mathcal{X} \rightarrow \mathbb{D}$ be a projective family over the unit disk, and let $\left(L, h_{L}\right)$ be a Hermitian line bundle, with the properties $(\mathrm{C})$ and $(\mathrm{R})$ above. We define

$$
\overline{\mathcal{I}_{0}^{(q)}}:=\overline{\mathcal{I}\left(h_{L \mid \mathcal{X}_{0}}\right)^{(q)}} .
$$

Then there exists a universal constant $C_{0}>0$ such that for any positive integers $p \geq q$ and for any section

$$
u \in H^{0}\left(\mathcal{X}_{0},\left(p K_{\mathcal{X}_{0}}+q L\right) \otimes \overline{\mathcal{I}_{0}^{(q)}}\right),
$$

there exists a section

$$
U \in H^{0}\left(\mathcal{X}, p K_{\mathcal{X}}+q L\right)
$$

such that

(i) over the central fiber we have $U_{\mid \mathcal{X}_{0}}=u \otimes d \pi^{\otimes p}$,

(ii) the next $L^{2 / p}$ integrability condition holds:

$$
\int_{\mathcal{X}}|U|^{\frac{2}{p}} e^{-\frac{q}{p} \varphi_{L}} \leq C_{0} \int_{\mathcal{X}_{0}}|u|^{\frac{2}{p}} e^{-\frac{q}{p} \varphi_{L}} .
$$

If $p=q=1$, then this is precisely the Ohsawa-Takegoshi extension theorem recalled above. For $p \geq 2$, the origins of the qualitative part of our result is the work of Siu. (See [29], [30], and also [6], [10], [12]-[17], [20]-[21], [22], [23], [27], [31]-[34], [36] for related statements.)

An important source of inspiration for Theorem 0.1 arises in particular from the results obtained by Tsuji [33], Takayama [31] and Hacon and McKernan [16] in connection with their work on pluricanonical series. To make this more transparent, we consider the following variant of Theorem 0.1.

Theorem $0.1^{\prime}$. Let $X$ be projective, and let $\left(L, h_{L}\right)$ be a Hermitian line bundle on $X$. Let $S \subset X$ be a nonsingular, irreducible submanifold of codimension 1 such that $h_{\mid S}$ is well defined. Assume that the next curvature condition is satisfied:

$$
\Theta_{h_{L}}(L) \geq \varepsilon_{0} \omega .
$$

Then any section of $\left(p K_{S}+q L\right) \otimes \overline{\mathcal{I}\left(h_{L \mid S}\right)^{(q)}}$ extends to $X$ as a section of the line bundle $p\left(K_{X}+S\right)+q L$.

This result is not a consequence of Theorem 0.1 but of its proof; the only difference is the version of the Ohsawa-Takegoshi theorem to be used in the inductive process. Moreover, following [21], it is possible to formulate (and 
prove) a higher codimensional analogue of the previous statement, where the hypersurface $S$ is replaced by a maximal center of some $\mathbb{Q}$-divisor.

We remark that in Theorem 0.1 it is not enough to assume the convergence of the integral $\int_{\mathcal{X}_{0}}|u|^{2 / p} e^{-(q / p) \varphi_{L}}$ in order to infer the extension of $u$; the additional hypothesis

$$
u \in \overline{\mathcal{I}_{0}^{(q)}}
$$

is needed. For many purposes, however, it is desirable to avoid this latter condition, that is, to replace it with something more "manageable." This is the problem we address in the second part of our article, where we assume for simplicity that $q=1$ and $\mathcal{I}\left(h_{L \mid \mathcal{X}_{0}}^{1 / p}\right)=\mathcal{O}_{\mathcal{X}_{0}}$. We notice that, in general, this latter hypothesis does not imply that $\mathcal{I}\left(h_{L \mid \mathcal{X}_{0}}\right)=\mathcal{O}_{\mathcal{X}_{0}}$.

We will analyze here the extension of sections of (2) under the hypothesis that the curvature current of $L$ is only assumed to be semipositive. Hence, unlike the usual setting, the bundle $L$ or its restriction to the central fiber is not necessarily big, but a natural vanishing assumption for the section to be extended is needed. Our next result can be seen as an effective version of the Ein-Popa theorem (see [12]); it is also a generalization of results due to Demailly [11] and Tsuji ([34], [35]). There are many hypotheses and cases of notation that we have to introduce before stating it, but they are natural in the context of the study of twisted pluricanonical systems on algebraic manifolds.

Let $\pi: \mathcal{X} \rightarrow \mathbb{D}$ be a proper, surjective map, where $\mathbb{D}$ is the unit disk. We assume that the central fiber $\mathcal{X}_{0}=\pi^{-1}(0)$ is nonsingular, and we let $L \rightarrow \mathcal{X}$ be a Hermitian line bundle such that $c_{1}(L)$ contains the current

$$
p([\Delta]+\alpha) \in c_{1}(L),
$$

where the notation is as follows.

(Eff) $\Delta:=\sum_{j \in J} \nu^{j} Y_{j}$ is an effective $\mathbb{Q}$-divisor, such that $p \nu^{j} \in \mathbb{Z}$ for any $j \in J$; moreover, we assume that the divisor $\Delta+\mathcal{X}_{0}$ has simple normal crossings.

(Diff) $\alpha$ is a closed, nonsingular, semipositive differential form of $(1,1)$ type, with the property that $\{p \alpha\} \in H^{2}(\mathcal{X}, \mathbb{Z})$.

Furthermore, we assume that the class $\left\{K_{\mathcal{X}}+1 / p L\right\}$ is pseudoeffective, and we let $h_{\min }$ be a metric with minimal singularities corresponding to it (for a complete presentation of this important concept, we refer to [11]); we denote by $\Theta_{\min }$ its curvature current. We assume that

$$
\nu_{\min }\left(\left\{K_{\mathcal{X}}+1 / p L\right\}, \mathcal{X}_{0}\right)=0
$$


that is, the minimal multiplicity of the class $\left\{K_{\mathcal{X}}+1 / p L\right\}$ along the central fiber $\mathcal{X}_{0}$ is equal to zero (see, e.g., [5]). Even under this additional hypothesis $(R e g)$, in general not all the sections of the bundle

$$
p K_{\mathcal{X}}+L_{\mid \mathcal{X}_{0}}
$$

admit an extension to $\mathcal{X}$ (see [13], [18]). Following [28] and [18], we define next an obstruction to extension divisor $\Xi$ on $\mathcal{X}_{0}$.

Let $A \rightarrow \mathcal{X}$ be an ample line bundle. The assumption (Reg) implies that the metric with minimal singularities $h_{\min , \varepsilon}$ corresponding to the class $K_{\mathcal{X}}+$ $1 / p L+\varepsilon A$ is not identically $+\infty$ when restricted to $\mathcal{X}_{0}$ (see [5]), so that we can decompose it according to the components of the restriction $\Delta_{\mid \mathcal{X}_{0}}$ as follows:

$$
\Theta_{\min , \varepsilon \mid \mathcal{X}_{0}}=\sum_{j \in J} \rho_{\min , \varepsilon}^{j}\left[Y_{j 0}\right]+\Lambda_{0 \varepsilon}
$$

where $Y_{j 0}:=Y_{j} \cap \mathcal{X}_{0}$ and where $\left(\rho_{\min , \varepsilon}^{j}\right)$ are positive real numbers. Here $\Lambda_{0 \varepsilon}$ is a closed positive current, whose Lelong number at the generic point of $Y_{j 0}$ is equal to zero, but of course, it may be singular along some other divisors or higher-dimensional analytic sets of $\mathcal{X}_{0}$. For each $j$, the sequence $\left(\rho_{\min , \varepsilon}^{j}\right)$ is decreasing, and we define

$$
\rho_{\min , \infty}^{j}:=\lim _{\varepsilon \rightarrow 0} \rho_{\min , \varepsilon}^{j}
$$

We introduce the notation

$$
J^{\prime}:=\left\{j \in J: \rho_{\min , \infty}^{j}<\nu^{j}\right\}
$$

and we assume, furthermore, that the next transversality condition is satisfied.

(Trans) We have $\nu^{j} \leq 1$, and for any subset $I \subset J^{\prime}$ and any $\varepsilon>0$, the restriction of the current $\Lambda_{0 \varepsilon}$ defined in (5) to the intersection $\bigcap_{m \in I} Y_{m} \cap \mathcal{X}_{0}$ is well defined.

Then we define the following effective $\mathbb{R}$-divisor:

$$
\Xi:=\sum_{j \in J^{\prime}} \rho_{\min , \infty}^{j} Y_{j}+\sum_{j \in J \backslash J^{\prime}} \nu^{j} Y_{j},
$$


and we observe that we have $\Xi \leq \Delta_{\mid \mathcal{X}_{0}}$. We denote by

$$
\varphi_{\Xi}:=\sum_{j \in J^{\prime}} \rho_{\min , \infty}^{j} \log \left|f_{Y_{j}}\right|^{2}+\sum_{j \in J \backslash J^{\prime}} \nu^{j} \log \left|f_{Y_{j}}\right|^{2}
$$

the local potential of the current $\Xi$, where for each $j$ the function $f_{Y_{j}}$ is a local equation of the hypersurface $Y_{j}$.

Let $h_{0}=e^{-\varphi_{0}}$ be a metric on the $\mathbb{Q}$-bundle $K_{\mathcal{X}_{0}}+1 / p L$ with the property that

$$
\Theta_{h_{0}}\left(K_{\mathcal{X}_{0}}+1 / p L\right) \geq 0
$$

and such that the following inequalities are satisfied:

$$
\begin{aligned}
\varphi_{0} & \leq \varphi_{\Xi} \\
\int_{\mathcal{X}_{0}} e^{\varphi_{0}-\frac{1}{p} \varphi_{L}} & <\infty .
\end{aligned}
$$

We denote by $\varphi_{L}$ the singular metric on $L$ induced by the decomposition (3). Relation (7) means that $\varphi_{0}$ is more singular than $\varphi_{\Xi}$. We state now our next result.

TheOREM 0.2. Let $\left(L, h_{L}\right)$ be a Hermitian line bundle whose Chern class contains the current $p([\Delta]+\alpha)$; we assume that $\Delta$ and $\alpha$ verify the hypotheses (Eff),(Trans), and (Diff). We also assume the existence of a metric $h_{0}=e^{-\varphi_{0}}$ on $K_{\mathcal{X}_{0}}+1 / p L$, with positive curvature current, and such that singularity and integrability assumptions (7) and (8) are satisfied.

Then the restriction $\varphi_{\min } \mid \mathcal{X}_{0}$ is well defined, and there exists a constant $C<0$ depending only on the quantity (8) and the geometry of the map $\pi$ such that the following inequality holds at each point of $\mathcal{X}_{0}$ :

$$
\varphi_{\min } \mid \mathcal{X}_{0} \geq C+\varphi_{0}
$$

If we assume, moreover, that $\nu^{j}<1$ for all $j$, then given any section $u$ of the bundle $p K_{\mathcal{X}_{0}}+L$ whose zero divisor is greater than $p \Xi$, there exists a section $U$ of $p K_{\mathcal{X}}+L$ extending $u$, and such that

$$
\int_{\mathcal{X}}|U|^{\frac{2}{p}} e^{-\frac{1}{p} \varphi_{L}} \leq C_{0} \int_{\mathcal{X}_{0}}|u|^{\frac{2}{p}} e^{-\frac{1}{p} \varphi_{L}} .
$$

Actually, we obtain an even more precise result: we can replace the metric $\varphi_{\min }$ in (9) with the so-called supercanonical metric on the $\mathbb{Q}$-bundle $K_{\mathcal{X} / \mathbb{D}}+$ 
$(1 / p) L$ (in the terminology of [11], [34]). We prefer, however, the formulation above, for reasons that will appear in a moment.

We remark that, as a consequence of (9), we obtain Ohsawa-Takegoshi type estimates for the extension $U$, provided that the section $u$ vanishes along the divisor $\Xi$.

If the form $\alpha$ in (Diff) is strictly positive, then the second part of the preceding result was established in [18] and [12]. Also, we refer to [11, Section 17] and the references therein for an enlightening introduction and related results around this circle of ideas.

In order to give another interpretation of Theorem 0.2 , we assume that we have $\nu^{j}<1$, that is, that the pair $(\mathcal{X}, \Delta)$ is klt in algebro-geometric language.

Let $L^{\prime}=L_{\mid \mathcal{X}_{0}} \otimes \mathcal{O}(-p \Xi)$; it is not too difficult to show that the class $\left\{K_{\mathcal{X}_{0}}+1 / p L^{\prime}\right\}$ is pseudoeffective (see, e.g., the arguments at the end of Section B). We denote by $\varphi_{\min }^{\prime}$ the metric with minimal singularities corresponding to $\left\{K_{\mathcal{X}_{0}}+1 / p L^{\prime}\right\}$; then we have

$$
\left|\varphi_{\min }\right| \mathcal{X}_{0}-\varphi_{\Xi}-\varphi_{\min }^{\prime} \mid \leq C,
$$

so the singularities of the restriction $\varphi_{\min \mid \mathcal{X}_{0}}$ are completely understood in terms of the extremal metric $\varphi_{\min }^{\prime}$. Except for the rationality of the coefficients $\rho_{\min , \infty}^{j}$, relation (10) is the metric version of the description of the restricted algebra in [18].

Furthermore, we show that inequality (9) of Theorem 0.2 has a compact counterpart, that is, when the couple $\left(\mathcal{X}, \mathcal{X}_{0}\right)$ is replaced by $(X, S)$, where we denote $S \subset X$ a nonsingular hypersurface of the projective manifold $X$. The bundle $L \rightarrow X$ is assumed to have properties (Eff),(Diff), and (Trans) above; in addition, we assume that we have

$$
\alpha \geq \gamma \Theta_{h}(\mathcal{O}(S))
$$

where $\gamma$ is a positive real number and $h$ is a nonsingular metric on the bundle $\mathcal{O}(S)$ associated to $S$.

The hypothesis concerning $\left\{K_{X}+S+(1 / p) L\right\}$, its corresponding minimal metric $\varphi_{\text {min }}$, and the metric $\varphi_{0}$ on $K_{X}+S+(1 / p) L_{\mid S}$ encoded in relations $(3)-(8)$ are assumed to hold transposed in the actual setting. In this case, the perfect analogue of the metric version of the "invariance of plurigenera" inequality (9) is true as follows. We have

$$
\varphi_{\min \mid S} \geq C+\varphi_{0}
$$


as it is shown by Theorem B.9. If we assume that $\nu^{j}<1$, then the analogue of inequality (11) in the present context can be rephrased as follows: a metric $\varphi_{0}$ on $K_{X}+S+(1 / p) L_{\mid S}$ is more singular than the restriction of $\varphi_{\min }$ to $S$ if and only if it satisfies relation ( 7$)$.

In Section $\mathrm{C}$ we prove an extension statement which was used in our previous work [4]; it is a quick consequence of the qualitative version of inequality (11) above (see Remark B.10).

As far as the organization of the present text is concerned, we mention here that Sections A and B can be followed independently (even if they share many similar techniques).

In conclusion, we believe that the metric point of view as initiated by Demailly [7] will be extremely useful for further research around the topics presented in this article.

\section{$\S$ A. Proof of Theorem 0.1}

We will first establish here the qualitative part of Theorem 0.1 ; the method we use is the "standard" one borrowed from the articles in the field quoted in the introduction. Nevertheless, there are quite a few things to be adapted to Theorem 0.1 , and therefore we will provide a complete treatment.

The main technical tool needed is the following effective extension theorem. Results of this kind first appeared in [26]; the version which is best adapted for what we need is taken from [30].

Theorem A.0 ([30, Theorem 2.1]). Let $\pi: \mathcal{X} \rightarrow \Delta$ be a projective family of smooth manifolds. Let $E \rightarrow \mathcal{X}$ be a line bundle, endowed with a (possibly singular) metric $h$, with semipositive curvature current. If $u \in H^{0}\left(\mathcal{X}_{0}, K_{\mathcal{X}_{0}}+\right.$ $E)$ is a section of the adjoint bundle of $E$ restricted to the central fiber such that

$$
\int_{\mathcal{X}_{0}}|u|^{2} e^{-\varphi}<\infty
$$

then there exists a section $U \in H^{0}\left(\mathcal{X}, K_{\mathcal{X}}+E\right)$ such that $U_{\mid \mathcal{X}_{0}}=u \wedge d \pi$, and moreover,

$$
\int_{\mathcal{X}}|U|^{2} e^{-\varphi} \leq C_{0} \int_{\mathcal{X}_{0}}|u|^{2} e^{-\varphi} .
$$

We recall the crucial fact that the constant $C_{0}$ above is absolutely universal. 
The above result shows that in order to extend some section $u$ of the bundle $p K_{\mathcal{X}_{0}}+q L$, it would be enough to get a metric on the bundle $(p-$ 1) $K_{\mathcal{X}}+q L$ such that $u$ is square-integrable with respect to the restriction of this metric to the central fiber. If $p=1$, then $q$ is equal to either zero or 1 ; if $q=0$, then we do not need any metric. If $q=1$, then one uses the metric of $L$.

For $p \geq 2$, the construction of the metric will be performed in the next two sections according to the following general principle. Let $F \rightarrow \mathcal{X}$ be a line bundle. We assume that for each $k \geq 1$ we are able to obtain a metric $h_{k}=e^{-\varphi_{k}}$ with semipositive curvature current on the $\mathbb{Q}$-bundle $(p-1) K_{\mathcal{X}}+$ $q L+(1 / k) F$ such that

- there exists a constant $C_{1}<0$ for which we have $\varphi_{k \mid \mathcal{X}_{0}} \geq C_{1}+((p-1) /$ p) $\log |u|^{2}$ pointwise on $\mathcal{X}_{0}$, for any $k \geq 1$;

- we also have $\sup _{k}\left(\sup _{\mathcal{X}} \varphi_{k}\right) \leq C_{2}$ for some positive constant $C_{2}$.

In this case, the metric we need in order to apply the $L^{2}$ extension Theorem A.0 is defined as a limit of $h_{k}$. The inductive process described in Sections A.1 and A.2 below shows that it is possible to fulfill this program, modulo the fact that for some technical reasons we will not have a single bundle $F$, but rather finitely many, as $k \rightarrow \infty$.

\section{A.1. Choice of the ample line bundle}

Let $u \in H^{0}\left(\mathcal{X}_{0}, p K_{\mathcal{X}_{0}}+q L\right)$ be the section we want to extend; we recall that by hypothesis we have

$$
u \in \overline{\mathcal{I}_{0}^{(q)}}:=\overline{\mathcal{I}\left(h_{L \mid \mathcal{X}_{0}}\right)^{(q)}}
$$

(this will play a crucial role in Section A.2). In order to start the inductive procedure which will construct our metric on the bundle $(p-1) K_{\mathcal{X}}+q L$, we first choose an ample line bundle on $\mathcal{X}$ with the following properties:

$\left(A_{0}\right)$ For each $\alpha=0, \ldots, p-q$, the bundle $\alpha K_{\mathcal{X}}+A$ is generated by global sections $\left(\tau_{i}^{(\alpha)}\right)$, where $i=1, \ldots, Q_{\alpha}$.

$\left(A_{1}\right)$ For each $\beta=1, \ldots, q$, the bundle $\beta A$ is globally generated by $\left(s_{j}^{(\beta)}\right)$, where $j=1, \ldots, N_{\beta}$.

$\left(A_{2}\right)$ Every section of $p K_{\mathcal{X}}+q L+(1+q) A_{\mid \mathcal{X}_{0}}$ extends to $\mathcal{X}$.

$\left(A_{3}\right)$ The sheaf $\mathcal{O}\left(K_{X}+L+A\right) \otimes \mathcal{I}\left(h_{\mid \mathcal{X}_{0}}\right)$ is generated by its global sections $\left(s^{(\gamma)}\right)$, for $\gamma=1, \ldots, M$.

Concerning the existence of such a line bundle, see, for example, [29]. We remark that $A$ will depend on $(p, q)$ because we impose the extension property $\left(A_{2}\right)$. 


\section{A.2. Inductive procedure}

We consider a triple of positive integers $(k, \beta, \alpha)$ such that $1 \leq \beta \leq q$ and such that $0 \leq \alpha \leq p-q$. In what will follow, we denote by $J$ a collection of elements of the set $\{1, \ldots, M\}$ (i.e., we allow repetitions among the elements of $J$ ), and we denote by $s^{(J)}:=\prod_{\rho \in J} s^{(\rho)}$ (we use the notation in the previous paragraph, $\left.A_{0}-A_{3}\right)$. The number of the elements of a collection $J$ (including repetitions) will be denoted by $|J|$.

In order to set up the inductive procedure, we introduce the next notation.

- If $\beta:=|J| \leq q-1$, then let

$$
u_{(j, i)}^{(k, J)} \in H^{0}\left(\mathcal{X}_{0}, k\left(p K_{\mathcal{X}}+q L\right)+\beta\left(K_{\mathcal{X}}+L+A\right)+(q-\beta) A+A_{\mid \mathcal{X}_{0}}\right)
$$

be the section defined by

$$
u_{(j, i)}^{(k, J)}:=u^{k} \otimes s^{(J)} \otimes s_{j}^{(q-\beta)} \otimes \tau_{i}^{(0)},
$$

where $i=1, \ldots, Q_{0}$ and $j=1, \ldots, N_{\beta}$. We remark that the above bundle is certainly not written in the most concise way; our intention is to make natural the tensor decomposition of the section $u_{(j, i)}^{(k, J)}$ in (12).

- If $|J|=q$, then let

$$
u_{(i)}^{(k, J, \alpha)} \in H^{0}\left(\mathcal{X}_{0}, k\left(p K_{\mathcal{X}}+q L\right)+q\left(K_{\mathcal{X}}+L+A\right)+\alpha K_{\mathcal{X}}+A_{\mid \mathcal{X}_{0}}\right)
$$

be the section defined by

$$
u_{(i)}^{(k, J, \alpha)}:=u^{k} \otimes s^{(J)} \otimes \tau_{i}^{(\alpha)},
$$

where $i=1, \ldots, Q_{\alpha}$.

We formulate the next property.

$\mathcal{P}(k, \beta, \alpha)$ : Given a triple $(k, \beta, \alpha)$ as above, for any collection $J \subset\{1, \ldots$, $q\}$ with $|J|=\beta$, we have the following.

- If $1 \leq|J| \leq q-1$, then for any $i, j$ as above there exists a section

$$
U_{(j, i)}^{(k, J)} \in H^{0}\left(\mathcal{X}, k\left(p K_{\mathcal{X}}+q L\right)+\beta\left(K_{\mathcal{X}}+L\right)+(1+q) A\right)
$$

whose restriction to the central fiber is equal to $u_{(j, i)}^{(k, J)}$.

- If $|J|=q$, then for any $i$ there exists a section

$$
U_{(i)}^{(k, J, \alpha)} \in H^{0}\left(\mathcal{X}, k\left(p K_{\mathcal{X}}+q L\right)+q\left(K_{\mathcal{X}}+L\right)+\alpha K_{\mathcal{X}}+(1+q) A\right)
$$

whose restriction to the central fiber is equal to $u_{(i)}^{(k, J, \alpha)}$. 
The core of the proof lies in the next statement (see [27, Proposition 2]).

Lemma A.2.1. The proposition $\mathcal{P}(k, \beta, \alpha)$ is true for any $k \in \mathbb{Z}_{+}$, any $1 \leq$ $\beta \leq q$, and any $0 \leq \alpha \leq p-q$. Moreover, the effective version of $\mathcal{P}(k, \beta, \alpha)$ holds. There exists a constant $C>0$ independent of $k$ such that if we denote by $h^{(k, \beta)}$ (resp., $\left.h^{(k, q, \alpha)}\right)$ the algebraic metric on the bundle $k\left(p K_{\mathcal{X}}+\right.$ $q L)+\beta\left(K_{\mathcal{X}}+L\right)+(1+q) A\left(\right.$ resp., on $k\left(p K_{\mathcal{X}}+q L\right)+q\left(K_{\mathcal{X}}+L\right)+\alpha K_{\mathcal{X}}+$ $(1+q) A$ ) induced by the family of sections $\left(U_{(j, i)}^{(k, J)}\right)_{i, j,|J|=\beta \leq q-1}$ (resp., by $\left.\left(U_{(i)}^{(k, J, \alpha)}\right)_{i,|J|=q}\right)$, then we have

$$
\max \left\{\int_{\mathcal{X}} e^{\varphi^{(k, \beta)}-\varphi^{(k, \beta-1)}-\varphi_{L}}, \int_{\mathcal{X}} e^{\varphi^{(k, 1)}-\varphi^{(k-1, q, p-q)}}\right\} \leq C
$$

and

$$
\max \left\{\int_{\mathcal{X}} e^{\varphi^{(k, q, \alpha)}-\varphi^{(k, q, \alpha-1)}}, \int_{\mathcal{X}} e^{\varphi^{(k, q, 0)}-\varphi^{(k-1, q-1)}-\varphi_{L}}\right\} \leq C .
$$

Proof. Even if the formulation of Lemma A.2.1 is somewhat complicated, the inductive procedure is quite natural, and it will be performed as follows. We first show that $\mathcal{P}(1,0,0)$ is valid, and we then prove that the next sequence of implications holds true:

$$
\begin{gathered}
\mathcal{P}(1,0,0) \rightarrow \mathcal{P}(1,1,0) \rightarrow \mathcal{P}(1,2,0) \rightarrow \cdots \rightarrow \mathcal{P}(1, q, 0) \rightarrow \\
\rightarrow \mathcal{P}(1, q, 1) \rightarrow \mathcal{P}(1, q, 2) \rightarrow \cdots \rightarrow \mathcal{P}(1, q, p-q) \rightarrow \mathcal{P}(2,1,0) \rightarrow \mathcal{P}(2,2,0) \rightarrow \cdots
\end{gathered}
$$

Notice that we allow $\beta$ to be equal to zero only for the first term in the previous sequence of implications; the reason is that $\varphi^{(k-1, q, p-q)}=\varphi^{(k, 0)}$. We remark that the proposition $\mathcal{P}(k, \beta, \alpha)$ is purely qualitative; nevertheless, the procedure we describe next will produce the uniform constant $C$ in the statement above as well.

Checking the first proposition $\mathcal{P}(1,0,0)$ is fairly easy: it is just the fact that $A$ is positive enough to satisfy the property $\left(A_{2}\right)$; this allows the extension of the sections $u \otimes s_{j}^{q} \otimes \tau_{i}^{(0)}$ for each $j=1, \ldots, N_{q}$ and $i=1, \ldots, Q_{0}$.

Assume now that for some indices $(k, \beta, \alpha)$ the property $\mathcal{P}(k, \beta, \alpha)$ has been established. Then we have to distinguish among several cases.

- We first consider the case $\alpha=0$ and $1 \leq \beta \leq q-1$. By property $\mathcal{P}(k, \beta$, $0)$, we deduce that for each index $(i, j, J)$ such that $1 \leq j \leq N_{q-\beta}, 1 \leq i \leq$ $Q_{0},|J|=\beta$, the section $u_{j, i}^{(k, J)}$ defined in (12) admits an extension

$$
U_{(j, i)}^{(k, J)} \in H^{0}\left(\mathcal{X}, k\left(p K_{\mathcal{X}}+q L\right)+\beta\left(K_{\mathcal{X}}+L\right)+(1+q) A\right) .
$$


Next we use the family of sections $\left(U_{j, i}^{(k, J)}\right)_{1 \leq j \leq N_{q-\beta}, 1 \leq i \leq Q_{0},|J|=\beta}$ to construct a metric $h^{(k, \beta)}$ on the bundle

$$
k\left(p K_{\mathcal{X}}+q L\right)+\beta\left(K_{\mathcal{X}}+L\right)+(1+q) A ;
$$

it will be singular in general, but its singularities over the central fiber are perfectly understood.

For each collection of integers $K$ such that $|K|=\beta+1$ and for each integer $i, j$, let us consider the section

$$
u_{j, i}^{(k, K)} \in H^{0}\left(\mathcal{X}_{0}, k\left(p K_{\mathcal{X}_{0}}+q L\right)+(1+\beta)\left(K_{\mathcal{X}_{0}}+L\right)+(1+q) A\right) ;
$$

we intend to extend it in an effective manner by the Ohsawa-Takegoshi theorem. To this end, we decompose the bundle above as

$$
\begin{aligned}
& k\left(p K_{\mathcal{X}}+q L\right)+(1+\beta)\left(K_{\mathcal{X}}+L\right)+(1+q) A=K_{\mathcal{X}}+k\left(p K_{\mathcal{X}_{0}}+q L\right) \\
& \quad+\beta\left(K_{\mathcal{X}_{0}}+L\right)+(1+q) A+L,
\end{aligned}
$$

and we remark that in this way it becomes the adjoint bundle of

$$
E:=k\left(p K_{\mathcal{X}}+q L\right)+\beta\left(K_{\mathcal{X}}+L\right)+(1+q) A+L .
$$

Now the bundle $E$ can be endowed with the metric $h^{(k, \beta)} \otimes h_{L}$; it is semipositively curved, and we check now the integrability of the section we want to extend with respect to it. We have the next relations:

$$
\begin{aligned}
I & :=\int_{\mathcal{X}_{0}}\left|u_{j, i}^{(k, K)}\right|^{2} e^{-\varphi^{(k, \beta)}-\varphi_{L}}=\int_{\mathcal{X}_{0}} \frac{\left|u_{j, i}^{(k, K)}\right|^{2}}{\sum_{l, m}^{|J|=\beta}\left|U_{l, m}^{(k, J)}\right|^{2}} e^{-\varphi_{L}} \\
& =\int_{\mathcal{X}_{0}} \frac{\left|u_{j, i}^{(k, K)}\right|^{2}}{\sum_{l, m}^{|J|=\beta}\left|u_{l, m}^{(k, J)}\right|^{2}} e^{-\varphi_{L}} \leq C \int_{\mathcal{X}_{0}} \frac{\left(\sum_{\gamma}\left|s^{(\gamma)}\right|^{2}\right)^{\beta+1}}{\left(\sum_{\gamma}\left|s^{(\gamma)}\right|^{2}\right)^{\beta}} e^{-\varphi_{L}-\varphi_{A}} \\
& \leq C .
\end{aligned}
$$

The second equality holds because of the definition of the metric $h^{(k, \beta)}$; the third one is given by the extension property $\mathcal{P}(k, \beta, 0)$. (Actually, here we use the fact that 0 is not a critical value of $\pi$ in order to identify $\left|u \wedge \pi^{\star}(d t)\right|^{2}$ with $|u|^{2}$.) The fourth inequality is obtained by simplification of the common factor $u^{k}$ and by the fact that all indices $J$ such that $|J|=\beta$ appear in the expression of the denominator. We also use the fact that the sections $\left(\tau_{i}^{(0)}\right)$ 
do not have common zeroes. Finally, the last inequality follows from the fact that the sections $s^{(\gamma)}$ belong to the multiplier ideal of the restriction of the metric $h_{L}$ to the central fiber.

The constant $C$ in the last line depends only on the auxiliary sections $\left(s^{(\gamma)}, s_{j}^{(m)}\right)$, and thus they are uniform with respect to $k$; also, $\varphi_{A}$ is just any smooth metric on $A$.

Thus, the requirements of the extension Theorem 0.1 are satisfied, and therefore, for each index $(K, i, j)$, we obtain

$$
U_{(j, i)}^{(k, K)} \in H^{0}\left(\mathcal{X}, k\left(p K_{\mathcal{X}}+q L\right)+(1+\beta)\left(K_{\mathcal{X}}+L\right)+(1+q) A\right)
$$

such that

(i) $U_{(j, i)}^{(k, K)}{ }_{\mid \mathcal{X}_{0}}=u_{(j, i)}^{(k, K)}$;

(ii) we have

$$
\int_{\mathcal{X}} e^{\varphi^{(k, \beta)}-\varphi^{(k, \beta-1)}-\varphi_{L}} \leq C
$$

for some constant $C$ which is a fixed multiple of one obtained a few lines above. Indeed, all we have to do is add the several estimates obtained above, and we remark that the number of the terms is bounded uniformly with respect to $k$.

Therefore, the first case is completely settled.

- We analyze here the second case, namely, that $\alpha=0$ and $\beta=q$; the arguments are quite similar to the previous case. Since we admit the validity of $\mathcal{P}(k, q, 0)$, we have the family of sections

$$
U_{(i)}^{(k, J)} \in H^{0}\left(\mathcal{X}, k\left(p K_{\mathcal{X}}+q L\right)+q\left(K_{\mathcal{X}}+L\right)+(1+q) A\right)
$$

such that

$$
U_{(i) \mid \mathcal{X}_{0}}^{(k, J)}=u_{(i)}^{(k, J)}
$$

as before, we can use them to define a metric $h^{(k, q)}$ on the bundle

$$
k\left(p K_{\mathcal{X}}+q L\right)+q\left(K_{\mathcal{X}}+L\right)+(1+q) A .
$$

We have to extend each member of the family of sections

$$
u_{(i)}^{(k, K, 1)} \in H^{0}\left(\mathcal{X}_{0}, k\left(p K_{\mathcal{X}}+q L\right)+q\left(K_{\mathcal{X}}+L\right)+K_{\mathcal{X}}+(1+q) A_{\mid \mathcal{X}_{0}}\right)
$$


where $|K|=q$. To this end we will again use the Ohsawa-Takegoshi theorem; we can write

$$
\begin{aligned}
& k\left(p K_{\mathcal{X}}+q L\right)+q\left(K_{\mathcal{X}}+L\right)+K_{\mathcal{X}}+(1+q) A=K_{\mathcal{X}}+k\left(p K_{\mathcal{X}}+q L\right) \\
& \quad+q\left(K_{\mathcal{X}}+L\right)+(1+q) A
\end{aligned}
$$

and we remark that in this way it becomes the adjoint bundle of

$$
E:=k\left(p K_{\mathcal{X}}+q L\right)+q\left(K_{\mathcal{X}}+L\right)+(1+q) A .
$$

The bundle $E$ can be endowed with the metric $h^{(k, q)}$; it is semipositively curved, and we now check the integrability of the section above:

$$
\begin{aligned}
I & :=\int_{\mathcal{X}_{0}}\left|u_{i}^{(k, K, 1)}\right|^{2} e^{-\varphi^{(k, q)}}=\int_{\mathcal{X}_{0}} \frac{\left|u_{i}^{(k, K, 1)}\right|^{2}}{\sum_{l, m}^{|J|=q}\left|U_{l, m}^{(k, J)}\right|^{2}} \\
& =\int_{\mathcal{X}_{0}} \frac{\left|u_{i}^{(k, K, 1)}\right|^{2}}{\sum_{l, m}^{|J|=q}\left|u_{l, m}^{(k, J)}\right|^{2}} \leq C \int_{\mathcal{X}_{0}} \frac{\left(\sum_{\gamma}\left|s^{(\gamma)}\right|^{2}\right)^{q}}{\left(\sum_{\gamma}\left|s^{(\gamma)}\right|^{2}\right)^{q}} d V \\
& \leq C .
\end{aligned}
$$

Thus, the second case is completely solved.

- The remaining cases we have to consider are $(1 \leq \alpha \leq p-q-1, \beta=q)$ and $(\alpha=p-q, \beta=q)$. We give the arguments only for the latter (and we leave the former to the interested reader). The implication we have to prove is that

$$
\mathcal{P}(k, q, p-q) \rightarrow \mathcal{P}(k+1,1,0) .
$$

Since the proposition $\mathcal{P}(k, q, p-q)$ is valid, we have the family of sections

$$
U_{(i)}^{(k, J, p-q)} \in H^{0}\left(\mathcal{X},(k+1)\left(p K_{\mathcal{X}}+q L\right)+(1+q) A\right)
$$

such that

$$
U_{(i) \mid \mathcal{X}_{0}}^{(k, J, p-q)}=u_{(i)}^{(k, J, p-q)}
$$

Let $h^{(k, q, p-q)}$ be the algebraic metric given by the sections $\left(U_{(i)}^{(k, J, p-q)}\right)$ above, where $|J|=q$ and $1 \leq i \leq Q_{p-q}$. Consider the section

$$
u_{(j, i)}^{(k+1, K)} \in H^{0}\left(\mathcal{X}_{0},(k+1)\left(p K_{\mathcal{X}_{0}}+q L\right)+K_{\mathcal{X}_{0}}+L+(1+q) A\right)
$$

(where $|K|=1$ ). We now check its integrability with respect to the metric $h^{(k, q, p-q)}$ twisted with the metric of $L$; in the next computations we skip 
some obvious steps which are direct consequences of the definition of the corresponding objects:

$$
\begin{aligned}
I: & =\int_{\mathcal{X}_{0}}\left|u_{(j, i)}^{(k+1, K)}\right|^{2} e^{-\varphi^{(k, q)}-\varphi_{L}} \\
& \leq C \int_{\mathcal{X}_{0}} \frac{\left|u \otimes s^{(K)} \otimes s_{j}^{(q-1)} \otimes \tau_{i}^{(0)}\right|^{2}}{\sum_{l,|J|=q}\left|s^{(J)} \otimes \tau_{l}^{(p-q)}\right|^{2}} \exp \left(-\varphi_{L}\right) \\
& \leq C .
\end{aligned}
$$

We remark that the last integral converges precisely because of the hypothesis $u \in \overline{\mathcal{I}_{0}^{(q)}}$. Indeed, we have

$$
|u|^{2} \leq C\left(\sum_{\gamma=1}^{M}\left|s^{(\gamma)}\right|^{2}\right)^{q}
$$

by the global generation property $\mathrm{A}_{3}$, hence, the inequality

$$
\begin{aligned}
& \int_{\mathcal{X}_{0}} \frac{\left|u \otimes s^{(K)} \otimes s_{j}^{(q-1)} \otimes \tau_{i}^{(0)}\right|^{2}}{\sum_{l,|J|=q}\left|s^{(J)} \otimes \tau_{l}^{(p-q)}\right|^{2}} \exp \left(-\varphi_{L}\right) \\
& \quad \leq C \int_{\mathcal{X}_{0}}\left|s^{(K)}\right|^{2} \exp \left(-\varphi_{L}\right)
\end{aligned}
$$

follows. This last integral is clearly convergent, again by $\mathrm{A}_{3}$. This ends the proof of the lemma.

Estimates (14) and (15) of Lemma A.2.1 show that we can consider the usual infimum construction metric

$$
h^{(\infty)}:=\liminf _{k} h^{(k, 1) \frac{1}{k}}
$$

of the bundle $p K_{\mathcal{X}}+q L$, which in addition has the following properties.

(a) The curvature current of $h^{(\infty)}$ is semipositive.

(b) The restriction of the metric $h^{(\infty)}$ to the central fiber is well defined, and we have $\sup _{\mathcal{X}_{0}}|u|_{h^{(\infty)}}<\infty$.

For the existence of the limit and the verification of the above relations, we refer, for example, to [11]. 
Now a last application of the Ohsawa-Takegoshi extension result will show that the section $u$ extends over the whole family. Indeed, we have

$$
p K_{\mathcal{X}}+q L=K_{\mathcal{X}}+\frac{p-1}{p}\left(p K_{\mathcal{X}}+q L\right)+\frac{q}{p} L,
$$

and we endow the bundle $((p-1) / p)\left(p K_{\mathcal{X}}+q L\right)+(q / p) L$ with the metric $\left(h^{(\infty)}\right)^{1-1 / p} \otimes h_{L}^{(q / p)}$. We have

$$
\int_{\mathcal{X}_{0}}|u|^{2} e^{-\frac{p-1}{p} \varphi^{(\infty)}-\frac{q}{p} \varphi_{L}} \leq C_{0} \int_{\mathcal{X}_{0}}|u|^{2 / p} e^{-\frac{q}{p} \varphi_{L}}<\infty,
$$

where the first inequality is given by property (b) above, and for the last one we use the fact that the coefficients of the section $u$ belong to the ideal $\overline{\mathcal{I}_{0}^{q}}$, together with the Hölder inequality. Again, we see that the condition $p \geq q$ is crucial.

This finishes the proof of Theorem 0.1 modulo the integrability of the appropriate root of the extension. To clarify this last point, we first show that

$$
\int_{\mathcal{X}} e^{\frac{\varphi^{(\infty)}-q \varphi_{L}}{p}}<\infty
$$

The relation above is obtained as follows: we multiply the inequalities (14) and (15) for successive parameters, and we use the Hölder inequality. We infer the existence of a positive constant $C$ such that

$$
\int_{\mathcal{X}} e^{\frac{\varphi^{(k+1,1)}-\varphi^{(1,1)}}{k p}-\frac{q}{p} \varphi_{L}} d \lambda \leq C
$$

for any $k \geq 1$.

Elementary properties of plurisubharmonic functions show that the sequence

$$
\frac{1}{k} \varphi^{(k, 1)}
$$

converges almost everywhere and in $L^{1}$ to the metric $\varphi^{(\infty)}$ (up to the choice of a subsequence). By Lebesgue's dominated convergence theorem, we can take the limit as $k \rightarrow \infty$ in (17) and obtain (16).

Now remember that the extension $U$ of our section $u$ satisfies the $L^{2}$ estimate

$$
\int_{\mathcal{X}}|U|^{2} e^{-\frac{p-1}{p} \varphi^{(\infty)}-\frac{q}{p} \varphi_{L}}<\infty
$$


Then we have

$$
\begin{aligned}
\int_{\mathcal{X}}|U|^{\frac{2}{p}} e^{-\frac{q}{p} \varphi_{L}} & =\int_{\mathcal{X}}|U|^{\frac{2}{p}} e^{-\frac{p-1}{p^{2}} \varphi^{(\infty)}-\frac{q}{p^{2}} \varphi_{L}} e^{\frac{p-1}{p^{2}} \varphi^{(\infty)}+\frac{(1-p) q}{p^{2}} \varphi_{L}} \\
& \leq\left(\int_{\mathcal{X}}|U|^{2} e^{-\frac{p-1}{p} \varphi^{(\infty)}-\frac{q}{p} \varphi_{L}}\right)^{\frac{1}{p}}\left(\int_{\mathcal{X}} e^{\frac{\varphi^{(\infty)}-q \varphi_{L}}{p}}\right)^{1-\frac{1}{p}}<\infty .
\end{aligned}
$$

As a conclusion to these considerations, we have found an extension $U$ of $u$ whose $L^{2 / p}$-norm is finite (see (18) above).

In the last part of this section, we establish the quantitative part of Theorem 0.1 , namely, the existence of a section

$$
U \in H^{0}\left(\mathcal{X}, p K_{\mathcal{X}}+q L\right)
$$

such that

(i) over the central fiber we have $U_{\mid \mathcal{X}_{0}}=u \wedge d \pi^{\otimes p}$;

(ii) the next $L^{1 / p}$ integrability condition holds:

$$
\int_{\mathcal{X}}|U|^{\frac{2}{p}} e^{-\frac{q}{p} \varphi_{L}} \leq C_{0} \int_{\mathcal{X}_{0}}|u|^{\frac{2}{p}} e^{-\frac{q}{p} \varphi_{L}} .
$$

Proof of (ii). We use basically the same arguments as in the proof of the $L^{2 / m}$ extension theorem in [4].

In the first place, we observe that the space of all the possible extensions of $u$ with integrable $L^{2 / p}$-seminorm is nonempty, thanks to (18) - this is the crucial point! Next we define $U$ to be an extension of $u$ which minimizes the previous seminorm; with this choice we now show that the estimate required in the theorem above is satisfied.

Indeed, let us consider the bundle

$$
p K_{\mathcal{X}}+q L=K_{\mathcal{X}}+\frac{p-1}{p}\left(p K_{\mathcal{X}}+q L\right)+\frac{q}{p} L ;
$$

it is the adjoint bundle of $((p-1) / p)\left(p K_{\mathcal{X}}+q L\right)+(q / p) L$, and we can endow the latter with the metric induced by the section $U$ raised to the power $1-1 / p$, twisted with the metric of $q / p L$. This metric has semipositive curvature and can be restricted to the central fiber, as is the case for the metric of $L$, and the section $U$ is not identically zero on $\mathcal{X}_{0}$.

The section $u \in H^{0}\left(\mathcal{X}_{0}, p K_{\mathcal{X}_{0}}+q L\right)$ is square-integrable with respect to the previous metric because the integrability condition reads as

$$
\int_{\mathcal{X}_{0}} \frac{|u|^{2}}{|u|^{2 \frac{p-1}{p}}} e^{-\frac{q}{p} \varphi_{L}}=\int_{\mathcal{X}_{0}}|u|^{\frac{2}{p}} e^{-\frac{q}{p} \varphi_{L}}<\infty .
$$


Here we use the fact that $U$ is an extension of $u$, as well as the hypothesis that $u$ belongs to the appropriate power of the multiplier ideal sheaf, which implies in particular that the last integral above is finite.

Thus, the Ohsawa-Takegoshi theorem shows the existence of some extension

$$
U_{1} \in H^{0}\left(\mathcal{X}, p K_{\mathcal{X}}+q L\right)
$$

of our section $u$ such that

$$
\int_{\mathcal{X}} \frac{\left|U_{1}\right|^{2}}{|U|^{2 \frac{p-1}{p}}} e^{-\frac{q}{p} \varphi_{L}} \leq C_{0} \int_{\mathcal{X}_{0}}|u|^{\frac{2}{p}} e^{-\frac{q}{p} \varphi_{L}} .
$$

But then we are done, since we necessarily have

$$
\int_{\mathcal{X}}|U|^{\frac{2}{p}} e^{-\frac{q}{p} \varphi_{L}} \leq \int_{\mathcal{X}} \frac{\left|U_{1}\right|^{2}}{|U|^{2 \frac{p-1}{p}}} e^{-\frac{q}{p} \varphi_{L}},
$$

because if not, the minimality property of the section $U$ will be violated. The argument is as follows. We assume that the inequality above does not hold; then we have

$$
\begin{aligned}
\int_{\mathcal{X}}\left|U_{1}\right|^{\frac{2}{p}} e^{-\frac{q}{p} \varphi_{L}} & =\int_{X} \frac{\left|U_{1}\right|^{\frac{2}{p}} e^{-\frac{q \varphi_{L}}{p^{2}}}}{|U|^{2 \frac{p-1}{p^{2}}}}|U|^{2 \frac{p-1}{p^{2}}} e^{-\frac{q}{p^{2}}(p-1) \varphi_{L}} \\
& \leq\left(\int_{X} \frac{\left|U_{1}\right|^{2}}{|U|^{2 \frac{p-1}{p}}} e^{-\frac{q}{p} \varphi_{L}}\right)^{\frac{1}{p}}\left(\int_{X}|U|^{\frac{2}{p}} e^{-\frac{q}{p} \varphi_{L}}\right)^{\frac{p-1}{p}} \\
& <\int_{X}|U|^{\frac{2}{p}} e^{-\frac{q}{p} \varphi_{L}} .
\end{aligned}
$$

The contradiction we have just obtained shows that Theorem 0.1 is completely proved.

\section{$\S B$. Canonical metrics and their restriction properties}

In this section we prove Theorem 0.2 and derive some of its consequences. To start with, we recall the general framework.

Let $\pi: \mathcal{X} \rightarrow \mathbb{D}$ be a proper, projective map, where $\mathbb{D}$ is the unit disk. We assume that zero is a regular value of $\pi$, and let $L \rightarrow \mathcal{X}$ be a Hermitian line bundle on $\mathcal{X}$, such that we have

$$
p([\Delta]+\alpha) \in c_{1}(L) .
$$


We assume that $\Delta$ and $\alpha$ satisfy the hypotheses (Eff) and (Diff), respectively; we also assume that the transversality condition (Trans) is fulfilled (hence, we tacitly assume that the requirement (Reg) holds true). We consider a metric $h_{0}=e^{-\varphi_{0}}$ on the $\mathbb{Q}$-bundle $K_{\mathcal{X}}+(1 / p) L_{\mid \mathcal{X}_{0}}$ with semipositive curvature current, which moreover satisfies the singularity and integrability assumptions (7) and (8) in the introduction. The conventions made in the introduction are in effect for the present paragraph.

Following Ein and Popa's (see [12]) elegant approach, for each $s=1, \ldots, p$, we define the set

$$
J_{s}:=\left\{j \in J^{\prime}: p \nu^{j} \geq s\right\}
$$

Then we can write

$$
p \sum_{j \in J^{\prime}} \nu^{j} Y_{j}=\sum_{s=1}^{p} \sum_{j \in J_{s}} Y_{j}
$$

and relation (20) induces a decomposition

$$
L=L_{1}+\cdots+L_{p-1}+L_{p}
$$

where $L_{s} \equiv \sum_{j \in J_{s}} Y_{j}$ for each $s=1, \ldots, p-1$, and such that $L_{p}$ admits a metric whose curvature form equals $p\left(\alpha+\sum_{j \in J \backslash J^{\prime}} \nu^{j}\left[Y_{j}\right]\right)+\sum_{j \in J_{p}}\left[Y_{j}\right]$.

Let $k \in \mathbb{Z}_{+}$, and let $r \in\{0,1, \ldots, p-1\}$; we introduce the notation

$$
L^{(r)}:=r K_{\mathcal{X}}+L_{1}+\cdots+L_{r}
$$

together with the convention that $L^{(0)}$ is the trivial bundle. By [29], there exists an ample line bundle $A$ on $\mathcal{X}$ having the following uniform global generation property: for any positively curved Hermitian bundle $\left(F, h_{F}\right)$ on the central fiber $\mathcal{X}_{0}$, the sheaf

$$
\mathcal{O}\left(\left(K_{\mathcal{X}_{0}}+F+L^{(r)}+A-\sum_{j \in J} Y_{j}\right) \otimes \mathcal{I}\left(h_{F}\right)\right)
$$

is generated by its global sections, for any $r=0, \ldots, p-1$. We also assume that $A$ is ample enough, so that the bundles $L^{(r)}+A$, their adjoints $K_{\mathcal{X}}+$ $L^{(r)}+A$, and $L^{(r)}+A-\sum_{j \in J} Y_{j}$ are very ample, for $r=0, \ldots, p$.

We introduce next the main technical tool which will lead us to Theorem 0.2 . 
For each $(k, r)$ within the range prescribed above, we will briefly recall the construction of the $k p$-Bergman metric on the bundle

$$
k\left(p K_{\mathcal{X} / \mathbb{D}}+L\right)+L^{(r)}+A
$$

where we denote by $K_{\mathcal{X} / \mathbb{D}}:=K_{\mathcal{X}}-p^{*} K_{\mathbb{D}}$ the relative canonical bundle of the map $\pi: \mathcal{X} \rightarrow \mathbb{D}$. The existence of this metric, together with its main features which are included in the next statement, is crucial for the proof of Theorem 0.2. We refer the reader to [3] and [4] for details and proofs (see also [34], [35], and the references therein for related results).

Let $\mathbb{D}^{\prime} \subset \mathbb{D}$ be a Zariski open set, such that for each $t \in \mathbb{D}^{\prime}$, each section of the bundle

$$
k\left(p K_{\mathcal{X}}+L\right)+L^{(r)}+A_{\mid \mathcal{X}_{t}}
$$

extends locally near $t$, for all $r=0, \ldots, p-1$. We recall next the construction of a metric $h_{\mathcal{X} / \mathbb{D}}^{(k p+r)}$ on this bundle, together with some of its relevant features. For any $t \in \mathbb{D}^{\prime}$, the restriction of the dual metric $h_{\mathcal{X} / \mathbb{D}}^{(k p+r) \star}$ to $\mathcal{X}_{t}$ is defined by

$$
|\xi|:=\sup _{u \in B_{t}^{k p}(1)}\left|\xi\left(\widetilde{u}_{x}\right)\right|,
$$

where $\xi$ is a vector in the dual bundle fiber $-k\left(p K_{\mathcal{X} / \mathbb{D}}+L\right)-L^{(r)}-A_{\mid \mathcal{X}_{t}, x}$. We denote by $B_{t}^{k p}(1)$ the set of all holomorphic sections $u$ of the bundle (23) restricted to $\mathcal{X}_{t}$ satisfying

$$
\int_{\mathcal{X}_{t}}|u|^{2 / k p} \exp \left(-\frac{\varphi_{r, A}}{k p}-\frac{\varphi_{L}}{p}\right) d \lambda \leq 1,
$$

and we denote $\widetilde{u}:=u \wedge d \pi^{\otimes k p}$. The metric $\varphi_{r, A}$ is nonsingular, positively curved on $L^{(r)}+A$, and $\varphi_{L}$ is induced by (19).

The result proved in [4] is as follows.

Theorem B.1 ([4, Corollary 4.2]). There exists a nonnegatively curved metric $h_{\mathcal{X} / \mathbb{D}}^{(k p+r)}$ on the bundle

$$
k\left(p K_{\mathcal{X} / \mathbb{D}}+L\right)+L^{(r)}+A
$$

such that

(a) when restricted to a fiber $\mathcal{X}_{t}$ with $t \in \mathbb{D}^{\prime}$, the metric $h_{\mathcal{X} / \mathbb{D}}^{(k p+r)}$ is induced by (23); 
(b) we let $h_{p}$ be a fixed, nonsingular metric on the bundle $p K_{\mathcal{X} / \mathbb{D}}+L$, and for each compact set $K \subset \Delta$ there exists a constant $C_{K}>0$ uniform with respect to $k$ such that

$$
C_{K} h_{\mathcal{X} / \mathbb{D}}^{(k p+r)} \geq h_{p}^{\otimes k} \otimes h_{r, A}
$$

on $K$.

An important observation is that the metric constructed above is not explicitly described on the set $\mathbb{D} \backslash \mathbb{D}^{\prime}$, so a priori we don't know the size of its singularities over that set. However, as we have remarked in [4], the "extendable sections" of the restriction

$$
k\left(p K_{\mathcal{X} / \mathbb{D}}+L\right)+L^{(r)}+A_{\mid \mathcal{X}_{t}}
$$

provide us with a lower bound for the weights of $h_{\mathcal{X} / \Delta}^{(k p+r)}$, even if $t \in \mathbb{D} \backslash \mathbb{D}^{\prime}$. We will explain this important (albeit elementary) fact next.

The main claim is the following. Let $\mu>0$ be a real number such that the disk centered at zero with radius $\mu$ does not contain any critical value of $\pi$, and let $\tau \in \mathbb{D}$ such that $|\tau|<\mu$. We consider a holomorphic section $U$ of the bundle $k\left(p K_{\mathcal{X} / \mathbb{D}}+L\right)+L^{(r)}+A$ over the whole family $\mathcal{X}$, whose global $L^{2 / k p}$-norm is finite; then (modulo an abuse of notation) we have

$$
\frac{|U(x)|^{2} e^{-\varphi_{\mathcal{X} / \mathbb{D}}^{(k p+r)}(x)}}{\left(\int_{\mathcal{X}_{\tau}}|U|^{2 / k p} \exp \left(-\frac{\varphi_{r, A}}{k p}-\frac{\varphi_{L}}{p}\right) d \lambda\right)^{k p}} \leq 1,
$$

where $x \in \mathcal{X}_{\tau}$ is an arbitrary point.

Indeed, if $\tau \in \mathbb{D}^{\prime}$, then the above claim is a consequence of the definition. If not, then we use a limit argument - since the weights $\varphi_{\mathcal{X} / \Delta}^{(k p+r)}$ are upper semicontinuous, and since the singularities of $1 / p \varphi_{L}$ are mild enough (see [4]).

We come back now to the metric $\varphi_{0}$ given by hypothesis, and we use it to define the space

$$
V_{k, r}:=\mathrm{H}^{0}\left(\mathcal{X}_{0},\left(k p K_{\mathcal{X}_{0}}+k L+L^{(r)}+A_{\mid \mathcal{X}_{0}}\right) \otimes \mathcal{I}\left(\psi_{k, r}\right)\right),
$$

where

$$
\psi_{k, r}:=(k p-1) \varphi_{0}+\sum_{j \in J}\left(1+\nu^{j}\right) \log \left|f_{Y_{j}}\right|^{2}+\widetilde{\varphi}_{r, A} .
$$


In the expression of the metric above, we denote by $\widetilde{\varphi}_{r, A}$ a nonsingular, positively curved metric on the bundle $L^{(r)}+A-\sum_{j \in J} Y_{j}$. We remark that we introduce an additional singularity $\left(1+\nu^{j}\right)$ instead of $\nu^{j}$ in the expression of the metric $\psi_{k, r}$; it will be useful during the proof of Lemma B.3.

The set $V_{k, r}$ is in fact a Hilbert space whose inner product is given by the formula

$$
\langle\langle u, v\rangle\rangle:=\int_{\mathcal{X}_{0}}\langle u, v\rangle e^{-\psi_{k, r}} .
$$

We consider an orthonormal basis $\left(u_{j}^{(k p+r)}\right)$ of $V_{k, r}$, and we prove next Lemmas B.2-B.5, that together will prove Theorem 0.2. The approach presented here has many similarities with and generalizes the ones in [11], [34], and [35].

Lemma B.2. There exists a constant $C$ independent of $k, j$ such that

$$
\int_{\mathcal{X}_{0}}\left|u_{j}^{(k p+r)}\right|^{2 / k p} \exp \left(-\frac{1}{p} \varphi_{L}-\frac{1}{k p} \varphi_{r, A}\right) \leq C
$$

for all $k \gg 0$.

Proof. This is a consequence of the Hölder inequality, as follows:

$$
\begin{aligned}
& \int_{\mathcal{X}_{0}}\left|u_{j}^{(k p+r)}\right|^{2 / k p} e^{-\frac{1}{p} \varphi_{L}-\frac{1}{k p} \varphi_{r, A}} \\
& \quad=\int_{\mathcal{X}_{0}}\left|u_{j}^{(k p+r)}\right|^{\frac{2}{k p}} e^{-\frac{k p-1}{k p} \varphi_{0}-\frac{1}{k p^{2}} \varphi_{L}-\frac{1}{k p} \varphi_{r, A}} e^{\frac{k p-1}{k p} \varphi_{0}-\frac{k p-1}{k p^{2}} \varphi_{L}} \\
& \quad \leq\left(\int_{\mathcal{X}_{0}}\left|u_{j}^{(k p+r)}\right|^{2} e^{(k p-1) \varphi_{0}-\frac{1}{p} \varphi_{L}-\varphi_{r, A}}\right)^{\frac{1}{k p}}\left(\int_{\mathcal{X}_{0}} e^{\varphi_{0}-\frac{1}{p} \varphi_{L}}\right)^{\frac{k p-1}{k p}} \\
& \quad \leq C .
\end{aligned}
$$

The last inequality is valid because of the integrability assumption (9) concerning the metric $\varphi_{L}$.

We introduce the set

$$
J^{1}:=\left\{j \in J: \nu^{j}=1\right\}
$$

and we show next that each element of $V_{k, r}$ admits an extension to $\mathcal{X}$ which vanishes along the divisor $\sum_{j \in J^{1}} Y_{j}$. This will be crucial for the study 
of $\varphi_{\mathcal{X} / \mathbb{D}}^{(k p+r)}$, given the inequality (24). Most of the "extension" arguments provided for the following lemma were developed in [29]; to our knowledge, their relevance in the actual context first appeared in [34].

Lemma B.3. For each $k, r$, and $j$ there exists a section

$$
U_{j}^{(k p+r)} \in H^{0}\left(\mathcal{X}, k p K_{\mathcal{X} / \mathbb{D}}+k L+L^{(r)}+A\right)
$$

whose restriction to $\mathcal{X}_{0}$ is equal to $u_{j}^{(k p+r)}$, and such that its zero divisor contains $\sum_{j \in J^{1}} Y_{j}$.

Proof. We use induction on $k p+r$; if $k=1$ and $r=0$, then the extension of the sections $u_{j}^{(p)}$ is a consequence of the ampleness of $A$, together with the fact that the $L^{2}$ condition in the definition of the space $V_{1,0}$ forces the vanishing of $u_{j}^{(p)}$ along $\sum_{j \in J} Y_{j}$.

Therefore, we assume that the extension of the sections $u_{j}^{(k p+r)}$ to $\mathcal{X}$ with the vanishing properties required by Lemma B.3 has already been shown to exist, and we let $u_{i}^{(k p+r+1)}$ be an element of the basis of $V_{k, r+1}$.

- If $r \leq p-2$, then we intend to use the global generation property of the bundle $A$ (see (22)), where the data are

$$
F:=(k p-1)\left(K_{\mathcal{X}_{\mid 0}}+\frac{1}{p} L\right)+\frac{1}{p} L+\sum_{j \in J} Y_{j}
$$

and $\varphi_{F}:=(k p-1) \varphi_{0}+\sum_{j \in J}\left(1+\nu^{j}\right) \log \left|f_{Y_{j}}\right|^{2}$. Since the section $u_{i}^{(k p+r+1)}$ belongs to the ideal associated to the metric $\varphi_{F}$, we have the pointwise inequality

$$
\left|u_{i}^{(k p+r+1)}\right|^{2} \leq C \sum_{j}\left|u_{j}^{(k p+r)}\right|^{2}
$$

by global generation property (22), where the norms are computed with respect to some nonsingular metric on the corresponding bundle.

We write the bundle $k p K_{\mathcal{X} / \mathbb{D}}+k L+L^{(r+1)}+A$ in adjoint form as follows:

$$
k p K_{\mathcal{X} / \mathbb{D}}+k L+L^{(r+1)}+A=K_{\mathcal{X} / \mathbb{D}}+L_{r+1}+k p K_{\mathcal{X} / \mathbb{D}}+k L+L^{(r)}+A .
$$

By the Ohsawa-Takegoshi theorem (Theorem A.0), in order to extend the section $u_{i}^{(k p+r+1)}$, it is enough to endow the bundle

$$
E:=L_{r+1}+k p K_{\mathcal{X} / \mathbb{D}}+k L+L^{(r)}+A
$$


with a semipositively curved metric, such that the $L^{2}$-norm of $u_{i}^{(k p+r+1)}$ with respect to it is finite. We denote by $\varphi_{L_{r+1}}$ the singular metric on $L_{r+1}$, whose curvature current is equal to $\sum_{j \in J_{r+1}}\left[Y_{j}\right]$, and by $\widetilde{\varphi}_{L_{r+1}}$ a nonsingular metric on this bundle, for which we cannot impose any curvature requirements. We also define the metric $h^{(k p+r)}$ on $k p K_{\mathcal{X} / \mathbb{D}}+k L+L^{(r)}+A$ induced by the family of sections $\left(U_{j}^{(k p+r)}\right)_{j}$.

For any parameters $\delta, \varepsilon, \tau \in \mathbb{R}_{+}$, we define the next metric on the bundle $E$ :

$$
\begin{aligned}
\varphi_{E}:= & (1-\delta) \varphi_{L_{r+1}}+\delta \widetilde{\varphi}_{L_{r+1}}+(1-\varepsilon) \varphi^{(k p+r)} \\
& +\varepsilon\left((k p-1) \varphi_{\min , \tau}+\frac{1}{p} \varphi_{L}+\widehat{\varphi}_{r, A}\right),
\end{aligned}
$$

where $\widehat{\varphi}_{r, A}$ is a positively curved nonsingular metric on the bundle $K_{\mathcal{X}}+$ $L^{(r)}+A$ and where $\varphi_{\min , \tau}$ is the metric induced on $K_{\mathcal{X}}+1 / p L$ by the metric with minimal singularities on $K_{\mathcal{X}}+1 / p L+\tau A$. We note that its curvature form is greater than $-\tau \omega_{A}$ and that its restriction to $\mathcal{X}_{0}$ has the expression in $(5)$.

We remark that $\left(E, e^{-\varphi_{E}}\right)$ is positively curved, provided that $\varepsilon \gg \delta$ and that $(k p-1) \tau \ll 1$. We still have to check that the following integral is convergent:

$$
\int_{\mathcal{X}_{0}}\left|u_{i}^{(k p+r+1)}\right|^{2} e^{-\varphi_{E}}<\infty .
$$

From relation (28), we see that the above $L^{2}$ condition is satisfied if we can show that

$$
\int_{\mathcal{X}_{0}}\left|u_{i}^{(k p+r+1)}\right|^{2 \varepsilon} e^{-(1-\delta) \varphi_{L_{r+1}}-\varepsilon(k p-1) \varphi_{\min , \tau}-\frac{\varepsilon}{p} \varphi_{L}}<\infty .
$$

(We ignore nonsingular weights in the expression of the $\varphi_{E}$.)

In order to establish relation (31), we recall that by hypothesis we have

$$
\varphi_{0} \leq \sum_{j \in J^{\prime}} \rho_{\min , \infty}^{j} \log \left|f_{j}\right|^{2}+\sum_{j \in J \backslash J^{\prime}} \nu^{j} \log \left|f_{j}\right|^{2} .
$$

Since the section $u_{i}^{(k p+r+1)}$ belongs to the space $V_{k, r+1}$, we infer that the divisor

$$
\sum_{j \in J^{\prime}}\left(\left[(k p-1) \rho_{\min , \infty}^{j}+\nu^{j}\right]+1\right) Y_{j}+\sum_{j \in J \backslash J^{\prime}}\left(k p \nu^{j}+1\right) Y_{j}
$$


is smaller than its zero divisor. Recall from the introduction that for any $\tau>0$, we have $\rho_{\min , \infty}^{j} \geq \rho_{\min , \tau}^{j}$, and therefore integral (31) is dominated by the quantity

$$
\int_{\mathcal{X}_{0}} \frac{e^{-(1-\delta) \varphi_{L_{r+1}}-\varepsilon(k p-1) \varphi_{\Lambda_{\tau 0}}} d \lambda}{\prod_{j \in J \backslash J^{\prime}}\left|f_{j}\right|^{2 \varepsilon\left(k p\left(\rho_{\min , \infty}^{j}-\nu^{j}\right)-\rho_{\min , \infty}^{j}-1\right)}} .
$$

Indeed, the lower bound of the vanishing of the section $u_{i}^{(k p+r+1)}$ as explained before is big enough to compensate for the singularities

$$
\varepsilon \sum_{j \in J^{\prime}}\left((k p-1) \rho_{\min , \infty}^{j}+\nu^{j}\right) \log \left|f_{Y_{j}}\right|^{2}
$$

arising from the restriction of $\varphi_{\min , \tau}$ and $(1 / p) \varphi_{L}$ to the central fiber; this is the main reason for introducing the additional singularities in the expression of the metric $\psi_{k, r}$.

We recall that we have $\varphi_{L_{r+1}}=\sum_{j \in J_{r+1} \subset J^{\prime}} \log \left|f_{j}\right|^{2}$, and hence the finiteness of the integral above is a consequence of the integrability Lemma B.12, which will be stated and proved at the end of the present section. Thus, all the hypotheses required by extension Theorem A.0 are fulfilled, so there exists a section $U_{i}^{(k p+r+1)}$ extending $u_{i}^{(k p+r+1)}$, and which is $L^{2}$ with respect to $\varphi_{E}$. We remark that by induction, we have

$$
\varphi^{(k p+r)} \leq \sum_{j \in J^{1}} \log \left|f_{Y_{j}}\right|^{2},
$$

and this is also the case for $(1 / p) \varphi_{L}$ (by definition), so we derive a similar conclusion for $\varphi_{E}$, given expression (29). Thus, the case $r \leq p-2$ is completely settled.

- We assume next that we have $r=p-1$. The section to be extended during this step is, say, $u_{i}^{(k p+p)} \in V_{k+1,0}$, so it verifies the $L^{2}$ condition

$$
\int_{\mathcal{X}_{0}}\left|u_{i}^{(k p+p)}\right|^{2} e^{-\psi_{k+1,0}}<\infty
$$

hence, we get

$$
\int_{\mathcal{X}_{0}} \frac{\left|u_{i}^{(k p+p)}\right|^{2}}{\prod_{j \in J \backslash J^{\prime}}\left|\sigma_{j}\right|^{2 p \nu^{j}}} e^{-\psi_{k, p-1}} d \lambda<\infty
$$


because of inequality (7); we denote by $\sigma_{j}$ the canonical section associated to the hypersurface $Y_{j}$. From the finiteness of the previous integral, we derive two conclusions. First, the section

$$
v^{(p)}:=\frac{u_{i}^{(k p+p)}}{\prod_{j \in J \backslash J^{\prime}} \sigma_{j}^{p \nu^{j}}}
$$

is holomorphic. Second, $v^{(p)}$ belongs to the multiplier ideal sheaf $\mathcal{I}\left(\psi_{k, p-1}\right)$. By global generation property (22) of the bundle $A$, we therefore obtain

$$
\frac{\left|u_{i}^{(k p+p)}\right|^{2}}{\prod_{j \in J \backslash J^{\prime}}\left|\sigma_{j}\right|^{2 p \nu^{j}}} \leq C \sum_{j}\left|u_{j}^{(k p+p-1)}\right|^{2} .
$$

Next, we write

$$
(k+1)\left(p K_{\mathcal{X} / \mathbb{D}}+L\right)+A=K_{\mathcal{X} / \mathbb{D}}+L_{p}+k p K_{\mathcal{X} / \mathbb{D}}+k L+L^{(p-1)}+A,
$$

so we consider the bundle

$$
E:=L_{p}+k p K_{\mathcal{X} / \mathbb{D}}+k L+L^{(p-1)}+A .
$$

In order to endow it with a metric, we recall that the Chern class of $L_{p}$ contains the current

$$
p\left(\alpha+\sum_{j \in J \backslash J^{\prime}} \nu^{j}\left[Y_{j}\right]\right)+\sum_{j \in J_{p}}\left[Y_{j}\right] .
$$

The metric whose associated curvature form is equal to the first term of the previous sum is denoted by $\varphi_{p}^{1}$, and we define $\varphi_{p}:=\sum_{j \in J_{p}} \log \left|f_{Y_{j}}\right|^{2}$. They induce a metric on the bundle $E$ as follows:

$$
\begin{aligned}
\varphi_{E}:= & \varphi_{p}^{1}+(1-\delta) \varphi_{p}+\delta \widetilde{\varphi}_{p}+(1-\varepsilon) \varphi^{(k p+p-1)} \\
& +\varepsilon\left((k p-1) \varphi_{\min , \tau}+\frac{1}{p} \varphi_{L}+\widetilde{\varphi}_{p-1, A}\right) .
\end{aligned}
$$

Its curvature current is positive as soon as $\delta \ll \varepsilon$ and $(k p-1) \tau \ll 1$.

The $L^{2}$-norm of the section $u_{i}^{(k p+p)}$ with respect to $\varphi_{E}$ is finite, provided that we have

$$
\int_{\mathcal{X}_{0}}\left|u_{j}^{(k p+p-1)}\right|^{2 \varepsilon} e^{-(1-\delta) \varphi_{L_{p}}-\varepsilon(k p-1) \varphi_{\min , \tau}-\frac{\varepsilon}{p} \varphi_{L}}<\infty
$$


for each $j$. As in the preceding case, the $L^{2}$ requirement reduces to (36) thanks to inequality (34) above. Inequality (36) was already established during the analysis of the preceding case (see (31)).

In conclusion, there exists an extension $U_{i}^{(k p+p)}$ of the section $u_{i}^{(k p+p)}$, which moreover is integrable with respect to $\varphi_{E}$. This implies that the section $U_{i}^{(k p+p)}$ vanishes as required in Lemma B.3, so the proof is complete.

The next statement is a summary of the preceding considerations.

Lemma B.4. We have

$$
\sup _{j}\left|u_{j}^{(k p)}(x)\right|^{\frac{2}{k p}} \leq C^{-1} e^{\frac{1}{k p} \varphi_{\mathcal{X} / \Delta}^{(k p)}(x)}
$$

for any $k$, as well as for any $x \in \mathcal{X}_{0}$.

Proof. Indeed, we specialize relation (24) for $\tau:=0$, and $U:=U_{j}^{(k p)}$ (see Lemma B.3); combined with Lemma B.2, it gives inequality (37) above.

As a consequence of the regularization theorem due to Demailly [8], we have the following very precise estimate.

Lemma B.5. There exists a constant $C$ such that we have

$$
(k p-1) \varphi_{0}(x)+\sum_{j \in J}\left(1+\nu^{j}\right) \log \left|f_{Y_{j}}\right|^{2} \leq C \log k+\log \sup _{j}\left|u_{j}^{(k p)}(x)\right|^{2}
$$

for any $x \in \mathcal{X}_{0}$ and $k \in \mathbb{Z}_{+}$large enough.

Proof. We refer the reader to [8] and [11]; the preceding inequality is obtained from the proof of the main theorem.

Proof of Theorem 0.2. By Theorem B.1(b), we infer the existence of a positively curved limit metric

$$
\varphi_{\mathcal{X} / \Delta}^{(\infty)}:=\lim \sup _{k} \frac{1}{k p} \varphi_{\mathcal{X} / \Delta}^{(k p)}
$$

on the $\mathbb{Q}$-bundle $K_{\mathcal{X} / \mathbb{D}}+1 / p L$. By Lemmas B.2-B.5, the metric $\varphi_{\mathcal{X} / \Delta}^{\infty}$ is less singular than $\varphi_{0}$ when restricted to the central fiber $\mathcal{X}_{0}$. This metric is clearly more singular than $\varphi_{\min }$, so inequality (9) of Theorem 0.2 is established. (The uniformity of the constant $C$ in (9) is obtained by inspection of the proof of Lemmas B.2-B.5.) 
REMARK B.6. The "traditional" method of proving this kind of results does not seem to work in this generalized setting. The reason is that we have to change the parameters $\delta, \varepsilon$ in the proof of Lemma B.3 as $k \rightarrow \infty$, and the usual "concavity of the log" (in [27], [30]) cannot be applied in order to obtain the estimates needed for the justification of the limit metric above. The asymptotic $k p$-Bergman metric somehow converts the qualitative information of Lemma B.3 into an effective estimate.

We state the second part of Theorem 0.2 as a separate corollary.

Corollary B.7. Let $u$ be a section of the bundle $p K_{\mathcal{X}_{0}}+L_{\mid \mathcal{X}_{0}}$, whose divisor of zeroes contains $p \sum_{j \in J^{\prime}} \rho_{\min , \infty}^{j} Y_{j 0}+p \sum_{j \in J \backslash J^{\prime}} \nu^{j} Y_{j 0}$; moreover, we assume that

$$
\int_{\mathcal{X}_{0}}|u|^{\frac{2}{p}} e^{-\frac{1}{p} \varphi_{L}}<\infty .
$$

Then there exists a section $U$ of $p K_{\mathcal{X}}+L$ extending $u$, and such that

$$
\int_{\mathcal{X}}|U|^{\frac{2}{p}} e^{-\frac{1}{p} \varphi_{L}} \leq C_{0} \int_{\mathcal{X}_{0}}|u|^{\frac{2}{p}} e^{-\frac{1}{p} \varphi_{L}} .
$$

This statement can be seen as a generalization of [12] and [18], where $L$ has an ample component. Certainly the convergence of the integral above just means that $u$ vanishes on the $\log$ canonical part of $(1 / p) L$, but we prefer this formulation because it is very well adapted for the study of similar results under more general boundaries $L$.

Proof. The vanishing properties of $u$ together with inequality (10) shows the existence of some constant $C$ such that we have

$$
|u|^{2} e^{-p \varphi_{\min }} \leq C<\infty
$$

on the central fiber. We have $p K_{\mathcal{X}}+L=K_{\mathcal{X}}+((p-1) / p)\left(p K_{\mathcal{X}}+L\right)+$ $(1 / p) L$, and the above inequality shows that the $L^{2}$-norm of $u$ with respect to the metric $(p-1) \varphi_{\min }+(1 / p) \varphi_{L}$ is finite. (Here we use the $L^{2 / p}$ convergence hypothesis (38) in Corollary B.7.) The proof ends thanks to the Ohsawa-Takegoshi theorem.

In order to prove inequality (10) stated in the introduction, we will assume that

$$
\nu^{j}<1
$$

for all $j \in J$. 
Let $L^{\prime}:=L_{\mid \mathcal{X}_{0}}-p \sum_{j \in J^{\prime}} \rho_{\min , \infty}^{j} Y_{j 0}-p \sum_{j \in J \backslash J^{\prime}} \nu^{j} Y_{j 0}$; by definition of the set $J^{\prime}$, we see that it is a pseudoeffective $\mathbb{R}$-divisor on the central fiber $\mathcal{X}_{0}$, whose adjoint

$$
p K_{\mathcal{X}_{0}}+L^{\prime}
$$

is pseudoeffective. This property is a consequence of the fact that $\varphi_{\min \mid \mathcal{X}_{0}}$ is well defined, so that we can write

$$
\Theta_{\min \mid \mathcal{X}_{0}}=\sum_{j \in J} \rho_{\min }^{j}\left[Y_{j 0}\right]+\Lambda_{0}
$$

the observation is that $\rho_{\min }^{j} \geq \rho_{\min , \varepsilon}^{j}$ for any $\varepsilon>0$, and thus the same inequality holds for the limit.

We denote by $\varphi_{\text {min }}^{\prime}$ a metric with minimal singularities corresponding to the bundle (40); a direct consequence of Theorem 0.2 is the next statement.

Corollary B.8. We have

$$
\left.\left|\varphi_{\min \mid \mathcal{X}_{0}}-\sum_{j \in J^{\prime}} \rho_{\min , \infty}^{j} \log \right| f_{j}\right|^{2}-\sum_{j \in J \backslash J^{\prime}} \nu^{j} \log \left|f_{j}\right|^{2}-\varphi_{\min }^{\prime} \mid \leq C
$$

pointwise on $\mathcal{X}_{0}$.

Proof. We first observe that the expression

$$
\psi:=\varphi_{\min \mid \mathcal{X}_{0}}-\sum_{j \in J^{\prime}} \rho_{\min , \infty}^{j} \log \left|f_{j}\right|^{2}-\sum_{j \in J \backslash J^{\prime}} \nu^{j} \log \left|f_{j}\right|^{2}
$$

corresponds to a positively curved metric of the bundle in (40) (despite the minus signs in its definition), and thus we have

$$
\psi \leq \varphi_{\min }^{\prime}+C
$$

by definition of the minimal metric associated to a cohomology class.

In the opposite sense, we note that we have

$$
\varphi_{\mathcal{X} / \mathbb{D} \mid \mathcal{X}_{0}}^{\infty} \geq \sum_{j \in J^{\prime}} \rho_{\min }^{j} \log \left|f_{j}\right|^{2}+\sum_{j \in J \backslash J^{\prime}} \nu^{j} \log \left|f_{j}\right|^{2}+\varphi_{\text {min }}^{\prime}+C
$$

by inequality (10), where the metric $\varphi_{0}$ corresponds to the right-hand side of the above relation. Finally, we clearly have

$$
\varphi_{\min } \geq \varphi_{\mathcal{X} / \mathbb{D}}^{\infty}
$$

and Corollary B.7 is proved. 
We turn now to the analysis of the compact version of Theorem 0.2. This means that we replace the couple $\left(\mathcal{X}, \mathcal{X}_{0}\right)$ by $(X, S)$, where $X$ be a projective manifold, and $S \subset X$ is a nonsingular hypersurface. We consider a line bundle $L \rightarrow X$ such that

$$
p([\Delta]+\alpha) \in c_{1}(L)
$$

where $\Delta=\sum_{j \in J} \nu^{j} Y_{j}$ and $\alpha \geq 0$ have the properties (Eff), (Diff), and (10a).

The class $\left\{K_{X}+S+1 / p L\right\}$ is assumed to be pseudoeffective, and we denote by $\varphi_{\min }$ a metric with minimal singularities corresponding to it; the associated curvature current will be denoted by $\Theta_{\min }$. As before, we suppose that

$$
\nu_{\min }\left(\left\{K_{X}+S+1 / p L\right\}, S\right)=0,
$$

and then we can define the quantities $\rho_{\min , \infty}^{j}$ exactly as in the previous case: let $\Theta_{\min , \varepsilon}$ be a current with minimal singularities within the class $\left\{K_{X}+S+1 / p L+\varepsilon A\right\}$; we have

$$
\Theta_{\min , \varepsilon \mid S}:=\sum_{j \in J} \rho_{\min , \varepsilon}^{j}\left[Y_{j \mid S}\right]+\Lambda_{S, \varepsilon}
$$

(thanks to assumption (44) above), where $\left(\rho_{\min , \varepsilon}^{j}\right)$ are positive real numbers and where $\Lambda_{S, \varepsilon}$ is a closed positive current defined on $S$. The limit of $\rho_{\min , \varepsilon}^{j}$ is denoted by $\rho_{\min , \infty}^{j}$. With this quantity, we define the set $J^{\prime} \subset J$ as in the introduction, and we assume that (Trans) holds as well.

Another part of the data is a positively curved metric $\varphi_{0}$ on the bundle $K_{X}+S+1 / p L_{\mid S}$; we assume that it satisfies properties (7) and (8) in the introduction. We discuss next the following version of Theorem 0.2.

THEOREM B.9. Under the hypothesis above, the metric $\varphi_{\min \mid S}$ is not identically $-\infty$, and we have

$$
\varphi_{\min \mid S} \geq C+\varphi_{0}
$$

pointwise on $S$.

Proof. The following arguments are completely similar to the ones provided for the proof of Theorem 0.2 along with the steps of Lemmas B.2-B.5. We will explain next the few things which are to be changed in order to conclude. 
- We consider the space

$$
V_{k, r}:=H^{0}\left(S,\left(k p\left(K_{X}+S\right)+k L+L^{(r)}+A_{\mid S}\right) \otimes \mathcal{I}\left(\psi_{k, r}\right)\right),
$$

where $\psi_{k, r}:=(k p-1) \varphi_{0}+\sum_{j \in J}\left(1+\nu^{j}\right) \log \left|f_{Y_{j}}\right|^{2}+\widetilde{\varphi}_{r, A}$ is a positively curved metric. Let $\left(u_{j}^{(k p+r)}\right)$ be an orthonormal basis of the space $V_{k, r}$; then we have

$$
\int_{S}\left|u_{j}^{(k p+r)}\right|^{2 / k p} \exp \left(-\frac{1}{p} \varphi_{L}-\frac{1}{k p} \varphi_{r, A}\right) \leq C
$$

for all $k \geq 1$ thanks to the Hölder inequality. (See Lemma B.2: in the expression under the integral sign, we identify $u_{j}^{(k p+r)}$ with a section of the bundle $k p K_{S}+k L+L^{(r)}+A_{\mid S}$.) Given the singularities of the metric $\psi_{k, r}$, we infer that the section $u_{j}^{(k p+r)}$ vanishes along the divisor $\sum_{m \in J} Y_{m \mid S}$.

- The algorithm used in the proof of Lemma B.3 shows that, given the integers $(k, r, j)$, the corresponding section $u_{j}^{(k p+r)}$ admits some extension to $X$. In fact, we show next that we can construct an extension of $u_{j}^{(k p+r)}$ which verifies an effective estimate, crucial for the rest of the proof.

Let $J^{1} \subset J$ be the set of indices $j \in J$ such that $\nu^{j}=1$. Exactly as in the proof of Lemma B.3, we show that there exists an extension $\widetilde{U}_{j}^{(k p+r)}$ of the section $u_{j}^{(k p+r)}$ which vanishes on the divisor $\sum_{j \in J^{1}} Y_{j}$. In particular, the section $\widetilde{U}_{j}^{(k p+r)}$ verifies the inequality

$$
\int_{X}\left|\widetilde{U}_{j}^{(k p+r)}\right|^{\frac{2}{k p}} \exp \left(-\frac{k \varphi_{L}+\varphi_{r, A}}{k p}-\varphi_{S}\right)<\infty .
$$

The next claim is that we can choose an extension $U_{j}^{(k p+r)}$ of the section $u_{j}^{(k p+r)}$ such that

$$
\begin{array}{r}
\int_{X}\left|U_{j}^{(k p+r)}\right|^{\frac{2}{k p}} \exp \left(-\frac{k \varphi_{L}+\varphi_{r, A}}{k p}-\varphi_{S}\right) \\
\leq C_{0} \int_{S}\left|u_{j}^{(k p+r)}\right|^{\frac{2}{k p}} \exp \left(-\frac{k \varphi_{L}+\varphi_{r, A}}{k p}\right)
\end{array}
$$

for all $k \gg 0$, where the constant $C_{0}$ is independent of $k$. Indeed, the proof of the $L^{p}$-Ohsawa-Takegoshi theorem in [4] shows that the extension of $u_{j}^{(k p+r)}$ which minimizes the left-hand side of (49) will automatically verify 
the estimates; we do not provide here any further details but rather remark that this is the only place in the proof where condition (10a) given in the introduction is used.

- We denote by $\psi^{(k p)}$ the metric on the bundle $k p\left(K_{X}+S\right)+k L+A$ associated to the set of sections $U_{j}^{(k p)}$. The inequality (49) above shows that

$$
\psi^{(\infty)}:=\lim _{\text {reg }} \sup _{k \rightarrow \infty} \frac{1}{k p} \psi^{(k p)}
$$

is a positively curved metric on the $\mathbb{Q}$-bundle $K_{X}+S+(1 / p) L$. Its restriction to $S$ is greater than $\varphi_{0}$ (up to a constant) by the same arguments as in Lemmas B.4 and B.5. Certainly the metric $\psi^{(\infty)}$ is smaller than the metric with minimal singularities, so that Corollary B.8 is proved.

Question. One of the important points in the proof of Corollary B.8 was that we can construct the extensions $U_{j}^{(k p)}$ vanishing along $\sum_{j \in J_{1}} Y_{j}$. We describe next a related question.

Let $\Delta$ be an effective divisor on $X$; one can define a notion of the $\Delta$ minimal metric on $K_{X}+S+(1 / p) L$, that is, the upper envelope of all normalized, positively curved metrics which are at least as singular as the quasi-plurisubharmonic function associated to the divisor $\Delta$. We denote this object by $\varphi_{\min , \Delta}$, and we assume that its restriction to $S$ is not identically $-\infty$. The question is how to identify the restriction $\varphi_{\min , \Delta \mid S} ;$ more precisely, we ask for a criterion similar to Corollary B.7. Unfortunately, the methods used and developed in this article do not seem to be very helpful in this direction.

REMARK B.10. A large part of the proof of Theorem B.9 can be applied in a more general setting, but it gives only a qualitative result.

For example, instead of the set $J^{\prime}$ used in the introduction, we can define

$$
J_{0}^{\prime}:=\left\{j \in J: \rho_{\min , \infty}^{j}<\nu^{j} \leq 1\right\}
$$

we also formulate the next condition.

$\left(\right.$ Trans $\left.^{\prime}\right)$ For any subset $I \subset J_{0}^{\prime}$ and for any $\varepsilon>0$, the restriction of the current $\Lambda_{0 \varepsilon}$ defined in relation (5) $\bigcap_{m \in I} Y_{m} \cap S$ is well defined.

The hypotheses are the same as for Theorem B.9, except that we use $J_{0}^{\prime}$ and $\left(\right.$ Trans $\left.{ }^{\prime}\right)$ to replace $J^{\prime}$ and (Trans), respectively. Then we infer the following result, which will be crucial for the next section. 
Consider the space $V_{k, r}$ as in (46); then relation (47) still holds without further modifications. Concerning the second bullet in the proof of Theorem B.9, we can only show the existence of an extension $U_{j}^{(k p+r)}$ of $u_{j}^{(k p+r)}$, without estimate (48). The family $\left(U_{j}^{(k p)}\right)_{j}$ defines a metric $\psi^{(k p)}$ on the bundle $k p\left(K_{X}+S\right)+k L+A$, and we have the estimate

$$
\psi^{(k p)} \geq(k p-1) \varphi_{0}+\sum_{j \in J}\left(1+\nu^{j}\right) \log \left|f_{Y_{j}}\right|^{2}+C
$$

pointwise on $S$. A last observation is that if the bundle $p\left(K_{X}+S\right)+L$ happens to be big, then we can define the space $V_{k, r}$ by using sections of $k p\left(K_{X}+S\right)+k L+L_{\mid S}^{(r)}$ (i.e., without the additional twisting with $A$ ) by a slight modification of the weight as in [11, Section 17]. Then the family of extensions $\left(U_{j}^{(k p)}\right)_{j}$ are sections of $k p\left(K_{X}+S\right)+k L+L^{(r)}$, and the above inequality becomes

$$
\psi^{(k p)} \geq\left(\left(k-k_{0}\right) p-1\right) \varphi_{0}+\psi+\sum_{j \in J}\left(1+\nu^{j}\right) \log \left|f_{Y_{j}}\right|^{2}+C,
$$

where $\psi$ is a metric on $k_{0} p\left(K_{X}+S\right)+k_{0} L$ whose curvature current is greater than a Kähler metric, and $k_{0}$ is a large enough integer.

A consequence of Theorem B.9 is that the norm with respect to the metric $e^{-\varphi_{\min \mid S}}$ of any section $u$ of the bundle $p\left(K_{X}+S\right)+L_{\mid S}$ whose zero set contains the divisor $\sum_{j \in J^{\prime}} \rho_{\min , \infty}^{j} Y_{j \mid S}+\sum_{j \in J \backslash J^{\prime}} \nu^{j} Y_{j \mid S}$ is uniformly bounded. We show in the next corollary that $u$ admits an extension to $X$ in the klt case.

Corollary B.11. In addition to the hypothesis in Theorem B.9, we assume that $\nu^{j}<1$ for all $j \in J$. Then any section

$$
u \in H^{0}\left(S, p\left(K_{X}+S\right)+L_{\mid S}\right)
$$

whose zero set contains the divisor $\sum_{j \in J^{\prime}} \rho_{\min }^{j} Y_{j \mid S}+\sum_{j \in J \backslash J^{\prime}} \nu^{j} Y_{j \mid S}$ admits an extension to $X$.

Proof. Let $A \rightarrow X$ be an ample enough line bundle such that

$$
u \otimes \sigma_{A}
$$

extends to $X$, where $\sigma_{A}$ is a nonzero section of $A$. We denote by $U_{A}$ the corresponding extension, and we consider the section

$$
u^{\otimes 2} \otimes \sigma_{A} \in H^{0}\left(S, 2 p\left(K_{X}+S\right)+2 L+A_{\mid S}\right) .
$$


We construct next an extension of $u^{\otimes 2} \otimes \sigma_{A}$ which is divisible by $U_{A}$; the quotient will be the desired extension of $u$.

To this end, we have the equality

$$
\begin{aligned}
2 p\left(K_{X}+S\right)+2 L+A= & K_{X}+S+p\left(K_{X}+S\right)+L+A \\
& +(p-1)\left(K_{X}+S+\frac{1}{p} L\right)+\frac{1}{p} L
\end{aligned}
$$

and for each positive $\varepsilon$, we consider the metric

$$
\log \left|U_{A}\right|^{2}+(p-1) \varphi_{\min }+\frac{1}{p} \varphi_{L}
$$

Its curvature form is semipositive, and it dominates $(\gamma / p) \Theta_{h}(\mathcal{O}(S))$. In order to apply [9], we have to check next the integrability condition

$$
\int_{S} \frac{\left|u^{2} \otimes \sigma_{A}\right|^{2}}{\left|u \otimes \sigma_{A}\right|^{2}} \exp \left(-(p-1) \varphi_{\min }-\frac{1}{p} \varphi_{L}\right)<\infty .
$$

This is, however, obvious, since by Corollary B.8 we have

$$
|u|^{2} \leq C e^{p \varphi_{\min \mid S}}
$$

at each point of $S$, and the restriction to $S$ of $e^{-1 / p \varphi_{L}}$ is convergent.

Thus, we obtain a section

$$
V \in H^{0}\left(X, 2 p\left(K_{X}+S\right)+2 L+A\right)
$$

whose restriction to $S$ is equal to $u^{2} \otimes \sigma_{A}$, and such that

$$
\int_{X} \frac{|V|^{2}}{\left|U_{A}\right|^{2}} \exp \left(-(p-1) \varphi_{\min }-\frac{1}{p} \varphi_{L}\right)<\infty .
$$

This in turn implies that the quotient $V / U_{A}$ is a holomorphic section of $p\left(K_{X}+S\right)+L$ and that it is equal to $u$ when restricted to $S$.

We establish next the following integrability criteria, which was used several times in this paragraph.

Lemma B.12. Let $\Theta$ be a closed $(1,1)$-current on a Kähler manifold $(X, \omega)$ such that

$$
\Theta \geq-C \omega
$$


for some positive constant $C$. Let $\left(Y_{j}\right)_{j=1, \ldots, r}$ be a finite set of hypersurfaces which are supposed to be nonsingular and to have simple normal crossings. Moreover, we assume that the restriction of $\Theta$ to the intersection $\bigcap_{i \in I} Y_{i}$ is well defined for any $I \subset\{1, \ldots, r\}$. Then there exists a positive $\varepsilon_{0}=\varepsilon_{0}(\{\Theta\}, C)$ depending only on the cohomology class of the current and on the lower bound $C$ such that, for any $\delta \in] 0,1]$ and $\varepsilon \leq \varepsilon_{0}$, we have

$$
\int_{(X, x)} \exp \left(-(1-\delta) \sum_{j=1}^{r} \log \left|f_{Y_{j}}\right|^{2}-\varepsilon \varphi_{\Theta}\right) d \Lambda<\infty
$$

at each point $x \in X$.

Proof. Let $x \in X$ be an arbitrary point; we can assume that $x \in Y_{1} \cap \cdots \cap$ $Y_{b}$ and $y \notin Y_{k}$ for some $b \leq r, k \geq b+1$. For each $p=1, \ldots, b$, we define the complete intersection

$$
\Xi_{p}:=Y_{1} \cap \cdots \cap Y_{p},
$$

and the Skoda integrability theorem implies that

$$
\int_{\left(\Xi_{b}, x\right)} \exp \left(-\varepsilon \varphi_{\Theta}\right) d \lambda<\infty
$$

for any $\varepsilon \leq \varepsilon_{0} \ll 1$. (We remark that here we use the hypothesis concerning the restriction of $\Theta$ to the sets $\Xi_{p}$.)

By the local version of the Ohsawa-Takegoshi theorem (see [9]), we can extend the constant function equal to 1 on $\Xi_{b}$ to a holomorphic function $f_{b-1} \in \mathcal{O}\left(\Xi_{b-1}, x\right)$ such that

$$
\int_{\left(\Xi_{b-1}, y\right)}\left|f_{b-1}\right|^{2} \exp \left(-(1-\delta) \log \left|f_{Y_{b}}\right|^{2}-\varepsilon \varphi_{\Theta}\right) d \lambda<\infty
$$

we repeat this procedure $b$ times until we get a function $f_{0} \in \mathcal{O}(X, x)$ such that

$$
\int_{(X, x)}\left|f_{0}\right|^{2} \exp \left(-(1-\delta) \sum_{j=1}^{r} \log \left|f_{Y_{j}}\right|^{2}-\varepsilon \varphi_{\Theta}\right) d \lambda<\infty .
$$

Since the function $f_{0}$ is constant equal to 1 in a open set centered at $y$ in $\Xi_{b}$, we are done, except for the uniformity of $\varepsilon_{0}$.

Indeed, that $\varepsilon_{0}$ depends only on the quantities in the above statement is a consequence of the fact that the Lelong numbers of closed positive currents on Kähler manifolds are bounded by the cohomology class of the current. 
As a side remark, one can see that the preceding statement holds true under the weaker assumption

$$
\nu_{\cap_{i \in I} Y_{i}}(\Theta)=0
$$

that is, we claim that Lemma B.12 is true if the generic Lelong number of $\Theta$ along all the intersections above is zero. Indeed, one can apply the regularization theorem stated in [8] combined with the Hölder inequality in order to derive the general result; we leave the details to the interested reader.

\section{$\S$ C. Further applications}

In this section we will prove an extension statement which was used in [4, Theorem B.1.2]. We first recall the general setup in [4] (and use the notation in that article).

Let $X$ be a normal projective variety, and let $\Delta$ be an effective Weil $\mathbb{Q}$-divisor on $X$ such that $K_{X}+\Delta$ is $\mathbb{Q}$-Cartier. We consider $W \subset X$ an exceptional center of $(X, \Delta)$; in other words, we assume that there exists a $\log$ resolution $\mu: X^{\prime} \rightarrow X$ of the pair $(X, \Delta)$ together with a decomposition of the inverse image of the $\mathbb{Q}$-divisor $K_{X}+\Delta$ as follows:

$$
\mu^{\star}\left(K_{X}+\Delta\right)=K_{X^{\prime}}+S+\Delta^{\prime}+R-\Xi,
$$

such that

- $S$ is an irreducible hypersurface, such that $W=\mu(S)$;

- $\Delta^{\prime}:=\sum_{j} a^{j} Y_{j}$, where $x_{0} \in \mu\left(Y_{j}\right)$ and $\left.a^{j} \in\right] 0,1[$;

- the divisor $R$ is effective, and a hypersurface $Y_{j}$ belongs to its support if either $x_{0} \notin \mu\left(Y_{j}\right)$ or $Y_{j} \cap S=\emptyset$ (so, in particular, the restriction $R_{\mid S}$ is $\mu_{\mid S}$-vertical);

- the divisor $\Xi$ is effective and $\mu$-contractible, and in addition, we assume that the support of the divisors of the right-hand side of formula (C1) has strictly normal crossings.

In general, the center $W$ is singular, and we will assume that the restriction map $\mu_{\mid S}: S \rightarrow W$ factors through the desingularization $g: W^{\prime} \rightarrow W$, so that we have

$$
\mu_{\mid S}=g \circ p
$$

where $p: S \rightarrow W^{\prime}$ is a surjective projective map. 
Before stating our next result, we introduce a last piece of notation. Let $A$ be an ample bundle on $X$, and let $F_{1}, \ldots, F_{k}$ be a set of smooth hypersurfaces of $W^{\prime}$ with strictly normal crossings such that there exist positive rational numbers $\left(\delta_{j}\right)$ for which the $\mathbb{Q}$-bundle

$$
g^{\star}(A)-\sum \delta^{j} F_{j}+\varepsilon K_{W^{\prime}}
$$

is semipositive (in a metric sense) for any $\varepsilon$ small enough, and such that $g\left(F_{j}\right) \subset W_{\text {sing }}$ for each $j$. Indeed, a set $\left(F_{j}\right)$ with the properties specified above does exist (see, e.g., [4]).

The family $\left(F_{j}\right)$ induces a decomposition of the divisor $\Xi$ as follows:

$$
\Xi=\Xi_{1}+\Xi_{2}
$$

where by definition $\Xi_{1}$ is the part of the divisor $\Xi$ whose support restricted to $S$ is mapped by $p$ into $\bigcup_{j} F_{j}$.

The result we will prove next is the following.

TheOREM C.1. Let $T$ be any closed positive $(1,1)$-current such that there exists a line bundle $E$ on $X$ with the property that $T \equiv \mu^{\star}(E)_{\mid S}+m\left(K_{S / W^{\prime}}+\right.$ $\left.\Delta_{\mid S}^{\prime}\right)$. Then we have

$$
T \geq m\left[\Xi_{2 \mid S}\right]
$$

in the sense of currents on $S$.

Proof. We start with a few reductions. By hypothesis (51), we infer the existence of a nonsingular and semipositive $(1,1)$-form $\alpha$ such that

$$
\alpha \equiv C\left(g^{\star}(A)-\sum_{i} \delta^{i} F_{i}\right)+m K_{W^{\prime}}
$$

where $C>0$ is a large enough constant. Therefore, we obtain

$$
T+C \sum_{i} \delta^{i}\left[p^{\star}\left(F_{i}\right)\right]+p^{\star}(\alpha) \equiv \mu^{\star}(E+C A)+m\left(K_{X^{\prime}}+S+\Delta^{\prime}\right)_{\mid S} .
$$

By definition of the decomposition $\Xi=\Xi_{1}+\Xi_{2}$, irreducible components of the divisors $\sum_{i} \delta^{i} p^{\star}\left(F_{i}\right)$ and, respectively, $\Xi_{2 \mid S}$, are disjoint. (Here we use the fact that $\mu$ is a log resolution.) Therefore, in order to prove Theorem C.1, it is enough to show that the current

$$
\Theta:=T+C \sum_{i} \delta^{i}\left[p^{\star}\left(F_{i}\right)\right]+p^{\star}(\alpha)
$$


verifies the inequality

$$
\Theta \geq m\left[\Xi_{2 \mid S}\right]
$$

in the sense of currents on $S$.

We show next that inequality (54) is a consequence of Remark B.10. By relation $(\mathrm{C} 1)$, we have

$$
\begin{aligned}
& \mu^{\star}\left(E+C A+m\left(K_{X}+\Delta\right)\right)+m \Xi \\
& \quad=\mu^{\star}(E+C A)+m\left(K_{X^{\prime}}+S+\Delta^{\prime}+R\right) .
\end{aligned}
$$

We can assume that $A$ is large enough such that $K_{X}+\Delta+(1 / m)(C A+E)$ is ample as well; then we claim that the metric with minimal singularities $\varphi_{\min }$ associated to the big class

$$
\frac{1}{m}\left\{\mu^{\star}\left(E+C A+m\left(K_{X}+\Delta\right)\right)+m \Xi\right\}
$$

has the same singularities as $\varphi \Xi$.

To see this, we first recall that, by Hartogs's principle, the zero divisor of any section of the bundle

$$
k\left(\mu^{\star}\left(E+A+m\left(K_{X}+\Delta\right)\right)+m \Xi\right)
$$

is greater than $k m \Xi$. (Indeed, Hartogs's principle is still valid even if $X$ may have singularities; the thing is that it is normal.) Since cohomology class (56) is big, the regularization of closed positive currents in [8] shows that the positively curved metric $e^{-\varphi_{\min }}$ can be approximated with holomorphic sections of the bundle

$$
k\left(\mu^{\star}\left(E+A+m\left(K_{X}+\Delta\right)\right)+\Xi\right)
$$

in a very precise way: there exists a (singular) metric $\psi$ on the bundle $k_{0}\left(\mu^{\star}\left(E+A+m\left(K_{X}+\Delta\right)\right)+\Xi\right)$ such that $\left(k-k_{0}\right) \varphi_{\min }+\psi$ is smaller than the metric induced by a family of holomorphic sections of the bundle above (see [8]). Because $k \rightarrow \infty$, we have proved our claim.

Let $\varphi_{\Theta+R}$ be the metric of the bundle

$$
K_{X^{\prime}}+S+\Delta^{\prime}+R+\frac{1}{m} \mu^{\star}(E+A)_{\mid S}
$$

whose curvature current is equal to $\Theta+[R]$. 
We apply the results obtained in Remark B.10 for $\varphi_{0}:=\varphi_{\Theta+R}$. The hypotheses needed for inequality (50a) to hold are satisfied given the structure of $\varphi_{\min }$ discussed above. Hence, despite the fact that in inequality (50a) we lack an upper bound for the algebraic metric $\psi^{(k p)}$, we can infer that the metric $\varphi_{\Theta+R}$ is more singular than $m \varphi_{\Xi_{2}}$. The proof of Theorem C.1 is finished.

\section{REFERENCES}

[1] B. Berndtsson, On the Ohsawa-Takegoshi extension theorem, Ann. Inst. Fourier (Grenoble) 46 (1996), 1083-1094.

[2] - Integral formulas and the Ohsawa-Takegoshi extension theorem, Sci. China, Ser. A 48 (2005), 61-73.

[3] B. Berndtsson and M. Păun, Bergman kernels and the pseudo-effectivity of the relative canonical bundles, Duke Math. J. 145 (2008), 341-378.

[4] - A Bergman kernel proof of the Kawamata subadjunction theorem, preprint, arXiv:0804.3884v2 [math.AG]

[5] S. Boucksom, Cônes positifs des variétés complexes compactes, Ph.D. dissertation, Institut Fourier, Grenoble, France, 2002.

[6] B. Claudon, Invariance for multiples of the twisted canonical bundle, Ann. Inst. Fourier (Grenoble) 57 (2007), 289-300.

[7] J.-P. Demailly, "Singular Hermitian metrics on positive line bundles" in Conference on Complex Algebraic Varieties (Bayreuth, 1990), Lecture Notes in Math. 1507, Springer, Berlin, 1992, 87-104.

[8] - Regularization of closed positive currents and intersection theory, J. Algebraic Geom. 1 (1992), 361-409.

[9] _ , "On the Ohsawa-Takegoshi-Manivel extension theorem" in Conference on Complex Analysis and Geometry (Paris, 1997), Progr. Math. 188, Birkhauser, Basel, 1999, 47-82.

[10] — "Kähler manifolds and transcendental techniques in algebraic geometry" in International Congress of Mathematicians, I, Eur. Math. Soc., Zurich, 2007, 153-186.

[11] - Analytic methods in algebraic geometry, preprint, 2009.

[12] L. Ein and M. Popa, Extension of sections via adjoint ideals, preprint, arXiv:0811.4290 [math.AG]

[13] T. de Fernex and C. D. Hacon, Deformations of canonical pairs and Fano varieties, J. Reine Angew. Math. 651 (2011), 97-126.

[14] - Rigidity properties of Fano varieties, preprint, arXiv:0911.0504 [math.AG]

[15] C. D. Hacon, Extension theorems and the existence of flips, lecture series at Oberwolfach Mathematical Institute, October 12-18, 2008.

[16] C. D. Hacon and J. McKernan, Boundedness of pluricanonical maps of varieties of general type, Invent. Math. 166 (2006), 1-25.

[17] - "Extension theorems and the existence of flips" in Flips for 3-Folds and 4Folds?, Oxford Lecture Ser. Math. Appl. 35, Oxford University Press, Oxford, 2007, $76-110$.

[18] - Existence of minimal models for varieties of log general type, II, J. Amer. Math. Soc. 23 (2010), 469-490. 
[19] Y. Kawamata, Deformation of canonical singularities, J. Amer. Math. Soc. 12 (1999), $85-92$.

[20] - "On the extension problem of pluricanonical forms" in Algebraic Geometry: Hirzebruch 70 (Warsaw, 1988), Contemp. Math. 241, Amer. Math. Soc., Providence, 1999, 193-207.

[21] D. Kim, $L^{2}$ extension of adjoint line bundle sections, Ann. Inst. Fourier (Grenoble) 60 (2010), 1435-1477.

[22] R. Lazarsfeld, Positivity in Algebraic Geometry, Ergeb. Math. Grenzgeb. 48, Springer, Berlin, 2004.

[23] M. Levine, Pluri-canonical divisors on Kähler manifolds, Invent. Math. 74 (1983), 293-303.

[24] T. Ohsawa, On the extension of $L^{2}$ holomorphic functions, VI, A limiting case, Contemp. Math. 332, Amer. Math. Soc., Providence, 2003, 235-239.

[25] - "Generalization of a precise $\mathrm{L}^{2}$ division theorem" in Complex Analysis in Several Variables (Kyoto, 2001), Adv. Stud. Pure Math. 42, Math. Soc. Japan, Tokyo, 2004, 249-261.

[26] T. Ohsawa and K. Takegoshi, On the extension of $L^{2}$ holomorphic functions, Math. Z. 195 (1987), 197-204.

[27] M. Păun, Siu's invariance of plurigenera: A one-tower proof, J. Diff. Geom. 76 (2007), 485-493.

[28] - Relative critical exponents, non-vanishing and metrics with minimal singularities, preprint, arXiv:0807.3109 [math.AG]

[29] Y.-T. Siu, Invariance of plurigenera, Invent. Math. 134 (1998), 661-673.

[30] , "Extension of twisted pluricanonical sections with plurisubharmonic weight and invariance of semipositively twisted plurigenera for manifolds not necessarily of general type" in Complex Geometry (Göttingen, 2000), Springer, Berlin, 2002, 223-277.

[31] S. Takayama, Pluricanonical systems on algebraic varieties of general type, Invent. Math. 165 (2006), 551-587.

[32] - On the invariance and lower semi-continuity of plurigenera of algebraic varieties, J. Algebraic Geom. 16 (2007), 1-18.

[33] H. Tsuji, Extension of log pluricanonical forms from subvarieties, preprint, arXiv:0709.2710v2 [math.AG]

[34] - Canonical singular Hermitian metrics on relative canonical bundles, preprint, arXiv:0704.0566v5 [math.AG]

[35] - Canonical volume forms on compact Kähler manifolds, preprint, arXiv:0707.0111v1 [math.AG]

[36] D. Varolin, A Takayama-type extension theorem, Compos. Math. 144 (2008), 522540 . 
Bo Berndtsson

Chalmers University of Technology

SE-412 96 Gothenburg

Sweden

bob@math . chalmers . se

Mihai Păun

Institut Élie Cartan de Nancy

Université Henri Poincaré Nancy 1

B.P. 70239

54506 Vandocuvre-lès-Nancy CEDEX

France

paun@iecn.u-nancy.fr 